\title{
Exposure Knowledge Impact on Regional Seismic Risk Assessment of Bridge Portfolios
}

\section{Andres Abarcaa ( $\square$ andres.abarca@iusspavia.it)}

University School for Advanced Studies (IUSS) of Pavia https://orcid.org/0000-0003-4011-1496

\section{Ricardo Monteiro}

University School for Advanced Studies (IUSS) of Pavia

Gerard J. O'Reillya

University School for Advanced Studies (IUSS) of Pavia

\section{Research Article}

Keywords: regional seismic risk, bridges, exposure, knowledge level, machine learning

Posted Date: January 25th, 2022

DOI: https://doi.org/10.21203/rs.3.rs-1286863/v1

License: (1) This work is licensed under a Creative Commons Attribution 4.0 International License.

Read Full License 
$3{ }^{a}$ University School for Advanced Studies (IUSS) of Pavia, Pavia, Italy

$4 \quad *$ Corresponding Author

5 Andres Abarca, andres.abarca@iusspavia.it, University School for Advanced Studies (IUSS) of Pavia, 6 Piazza della Vittoria n.15, 27100 Pavia (Italy)

ORCID Information

9 Andres Abarca (0000-0003-4011-1496)

10 Ricardo Monteiro (0000-0002-2505-2996)

11 Gerard J. O'Reilly (0000-0001-5497-030X)

Abstract

The lack of structural information on existing bridges is a common problem faced by engineers when performing regional seismic risk assessment of large bridge portfolios. In most regions, the bridge inventory is composed of structures built over decades and detailed structural information of the existing configurations is difficult to obtain and can be expensive to survey. Most of the regional risk studies for bridges are done with incomplete exposure knowledge and usually rely on macro taxonomy-based approaches that average fragility information of assets with similar configurations. This leads to an unknown level of uncertainty in the results that is commonly not quantified or accurately communicated to the stakeholders. Accordingly, there is a need for a better understanding on how much uncertainty can be expected in results using such approaches, as well as for recommendations to those dealing with this type of project to define an appropriate required minimum knowledge of the inventory to obtain reasonable results. In this study, the seismic risk assessment of a portfolio of 617 bridges with complete structural information was performed and its results were used as a benchmark to quantify the expected uncertainty when considering different knowledge levels using a taxonomy-based approach as well as a machine learning model. The obtained results suggest that having detailed information on at least one third of the portfolio leads to a considerable reduction in uncertainty and that machine learning models can outperform traditional taxonomy-based methodologies only when a considerable level of knowledge of the inventory is available. 


\section{Introduction}

Regional seismic assessment of bridge portfolios has gained popularity as a tool for stakeholders and decision makers to quantify the risk associated with earthquake activity on their inventories. While the specific methodologies for this type of assessment vary, the overarching philosophy relies on dividing the problem into the components of hazard, exposure and vulnerability, which are later convoluted in a probabilistic fashion to estimate the annual rates of exceeding specific thresholds of structural performance (e.g., pier shear failure) or economic losses associated with repairing the damaged bridge structure.

Given this probabilistic nature of risk assessment, uncertainty plays a key role in the process. Two main types of uncertainty are generally recognized in risk assessment: aleatory uncertainty, which refers to the inherent random effects present in natural phenomena and therefore cannot be reduced; and epistemic uncertainty, which refers to the lack of knowledge associated with each component of risk. In the case of the exposure component, which deals with the number and characteristics of the physical assets included in the assessment, lack of structural information in terms of geometrical dimensions of elements, material properties or structural component configurations constitute a source of epistemic uncertainty that ultimately affects the accuracy of the overall risk assessment results. While it is possible to reduce this uncertainty by performing data collection and surveying campaigns, these require a large effort, time and cost, causing most practical seismic risk assessment applications for both bridges and buildings to be carried out with incomplete information.

Common practice when addressing the lack of specific structure-level information is to use macro taxonomy-based approaches that average fragility information of assets with similar configurations, which are expected to have similar performance or observed damage when subjected to equal levels of seismic demands. Such practice is the basis for the HAZUS (F.E.M.A., 2013) and SYNER-G (Pitilakis et al., 2014) methodologies, for example. In order to do this, taxonomy branches are defined by grouping key structural parameters that are assumed to influence structural capacity and seismic response. Subsequently, multiple representative structures within each taxonomy branch are analysed in detail with the intention of capturing the variability of the behaviour in each class and defining an average fragility curve. Such a curve can then be applied to each element in the inventory that have been identified as members of the class without the necessity of performing specific analysis for every individual asset.

While this practice is frequent and generally recommended for the regional assessment of buildings (D'Ayala et al., 2015), a recent study conducted on bridges (Stefanidou \& Kappos, 2019) concluded that the use of taxonomy-based fragility curves can significantly affect the accuracy of predictions for individual assets in a portfolio. Another study (Abarca et al., 2021) performed on an inventory of bridges with full information, confirmed their inaccuracy in bridge specific predictions, while also concluding, nevertheless, that the use of taxonomy-based curves leads to accurate estimates of the total direct losses for the entire portfolio. This means that these types of curves can potentially be a good alternative to assess aggregated losses over entire inventories if enough representative structures are used to accurately capture the variability within each taxonomy branch.

The number of structures that should be included in the detailed analysis to fully represent a taxonomy branch will depend on the classification scheme that is used to define the branches and the inherent variability in behaviour present in each resulting branch. Much debate exists on the appropriate bridge parameters to use to group bridge classes. While HAZUS is very popular (F.E.M.A., 2013), as evidenced by recent research (Mangalathu et al., 2017; Nielson \& DesRoches, 2007) and regularly used in risk projects worldwide (Chen et al., 2013; Yue et al., 2010; Nielson \& DesRoches, 2007), other options have been proposed (Mangalathu et al., 2017; Joint Research Centre, 2013) and researchers typically define their own classification depending on the specific characteristics of the inventory to be analyzed. Furthermore, since the inherent variability present in each classification is not known beforehand, the 
number of structures chosen to represent each branch is typically defined arbitrarily depending on the amount of information available for each specific case and inventory. All of this leads to an unknown level of uncertainty in the accuracy of the results obtained.

In the current study, a database of 308 existing bridges from the Italian road network is used to define multiple realizations and form a case study of 617 bridges located in the province of Salerno. This portfolio is used to evaluate the uncertainty that can be expected in the total direct economic losses calculated over the entire portfolio of each case study realization when increasing portions of the inventory are known. The intention is to provide researchers and practitioners dealing with seismic risk assessment of bridge inventories with a better understanding of the uncertainty level surrounding the results obtained when a fixed percentage of their inventory has full information available. This information can also guide the early stages of the regional assessment process, in defining the number of structures that should be properly surveyed and analysed to obtain a desired level of accuracy in direct loss calculations.

Furthermore, recent developments in machine learning applications to risk assessment projects have been shown to be promising to increase the accuracy in predictions of earthquake-related damage and losses. Mangalathu et al. (2019) tested the performance of multiple algorithms to predict the limit state of bridges in a portfolio following a seismic event based on specific bridge attributes. They concluded that the use of these algorithms allows for the increase in damage detection accuracy by incorporating multiple parameters in the calculation other than just the intensity measure level used in typical fragility curves. Another recent study (Kalakonas \& Silva, 2021) evaluated the use of artificial neural networks for the derivation of seismic vulnerability models for building portfolios and observed an overwhelming improvement in the reliability and accuracy in risk assessment predictions when compared to the traditional regression models, further highlighting their potential for risk assessment applications.

Also, the evaluation of the exposure knowledge impact when using a taxonomy-based approach is presented here. It is then used to train a machine learning model with the non-linear time-history analysis results of the known portions of the inventory and to subsequently estimate the fragility curves of each unknown asset. The results obtained using these predicted curves are also evaluated in terms of the uncertainty in the total direct economic losses calculated over the entire portfolio and compared with the results obtained using the traditional taxonomy-based approach.

\section{Methodology}

The methodology defined for this study, depicted graphically in Figure 1, initially consists of creating multiple networks with different configurations of bridges located in a case-study region. This is done by taking the assets from a portfolio of real bridges with fully known information and randomly locating them within an existing road network, thus creating multiple synthetic case study realisations. Each bridge is then analysed in its respective location in each case-study realisation according to detailed risk assessment procedures, enabling the determination of the direct economic losses (average annual losses, AAL) for the entire portfolio, which serves as a benchmark to evaluate the uncertainty that can be expected when incomplete information of the inventory is available. 


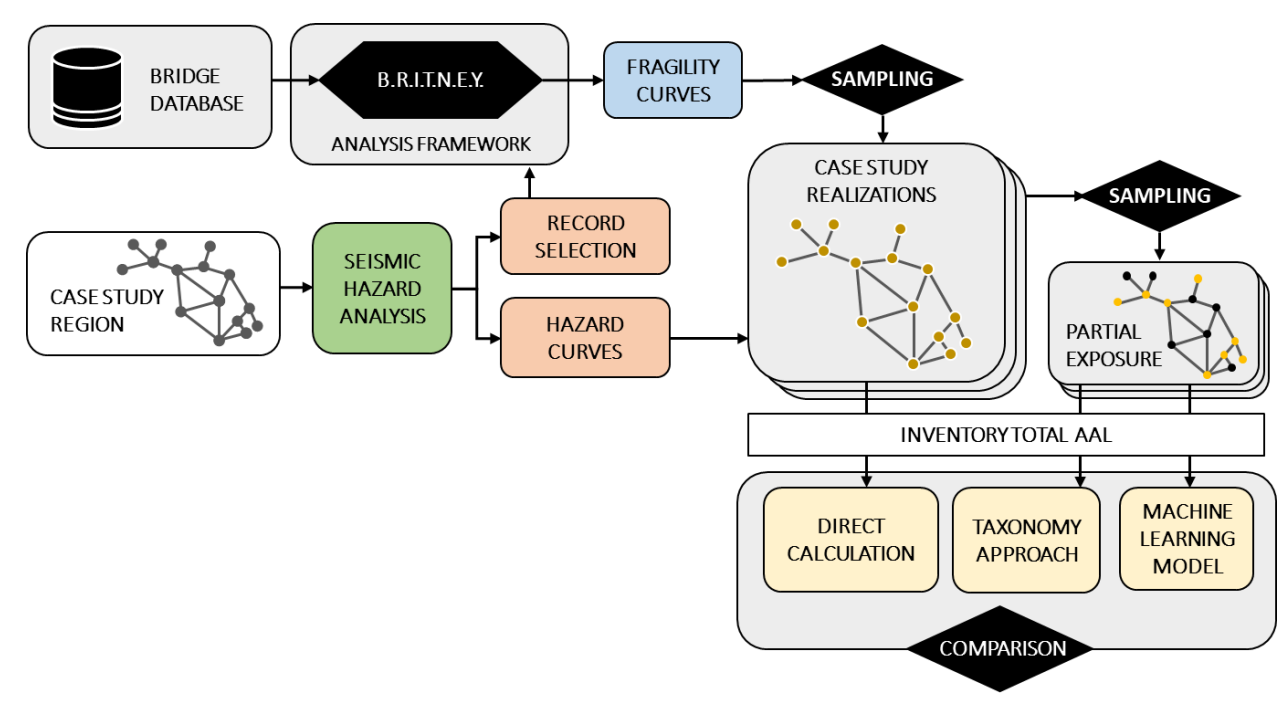

Figure 1: Methodology used to evaluate the effect for exposure knowledge for bridge inventories

A database containing 308 bridges from the National Autonomous Roads Corporation ANAS (Azienda Nazionale Autonoma delle Strade) inventory, collected and managed by the Eucentre Foundation (Borzi et al., 2015), was used to populate the bridge locations of the primary and secondary road networks of the Italian province of Salerno, using information taken from OpenStreetMap repositories (OpenStreetMap contributors, 2020). Once the case study portfolio distribution of bridges was created, a probabilistic seismic hazard analysis was carried out for the location of each bridge to determine hazard curves specific to each site. Furthermore, all bridges were grouped into four hazard zones, for which a conditional spectrum record selection was carried out using an automated tool (Ozsarac et al., 2021) considering two possible soil conditions (soft and stiff) to obtain ground motion record sets for each zone. These sets of 30 bi-directional earthquake records are conditioned on AvgSa, which is an intensity measure (IM) recently shown (Abarca et al., 2021; O'Reilly, 2021) to be quite advantageous when assessing multiple bridge structures compared to other IMs like PGA, PGV or Sa(T) commonly used. The AvgSa for this case was defined with a period range of $0.1 \mathrm{~s}$ to $1.7 \mathrm{~s}$ and spacing of $0.1 \mathrm{~s}$, and was used to condition the record selection for nine return periods of ground shaking, ranging from 98 to 9975 years.

Numerical models were created for each bridge using the BRI.T.N.E.Y (BRIdge auTomatic Nonlinear analysis based Earthquake fragilitY)modelling tool developed by Borzi et al. (2015). These were analysed via nonlinear time-history analysis (NLTHA) using the ground motion record sets selected for each asset location and fragility curves for the collapse limit state of each case-study bridge were determined. These fragility curves were then integrated with the hazard curves of each site to obtain the annual probability of collapse of each bridge. This was then multiplied by an estimate of the replacement cost of the bridge to determine their collapse-based AAL. A sampling process was then adopted, whereby portions of the database of results were randomly removed and the remaining ones used to calculate taxonomy-based fragility curves, which were then assigned to each asset of the realisations with removed information. Exposure rates between 5\% and $100 \%$, defined as the ratio between the number of assets with complete information and the total number of assets in the inventory, were evaluated by performing 40 different random samples for each exposure rate to capture the uncertainty in the calculation of total AAL using the taxonomy-based curves on the assets with incomplete information.

30 Additionally, the same sampling process was repeated but the known portions of the inventory at each 31 iteration were used to train a machine learning model for each bridge class to predict the collapse probability at specific IM levels for the bridges with incomplete information based on simple geometric properties of the actual structures. These predictions were then used to determine continuous fragility functions for each asset with incomplete information and estimate the total AAL of the entire inventory at 
each iteration. Finally, statistical trends of the uncertainty associated with each exposure rate when applying both the taxonomy-based approach and the machine learning model were defined and compared to determine the relative performance of each method. Recommendations are also provided on which method to use, depending on the percentage of known information and corresponding accuracy.

\section{3. Exposure model: case-study bridge inventory}

\section{6}

\subsection{Bridge database description}

As mentioned previously, a bridge database comprising 308 bridges was considered to create the case study for this research. These bridges form a part of the primary highway grid of the Italian road network and their actual geographic location is widely scattered across the country, as shown in Figure 2. The information considered in the database comprises a complete account of geometrical and structural properties of the bridges, allowing detailed structural numerical models of each asset to be created. Each asset in the database is a reinforced concrete (RC) bridge with two or more spans; a predominant configuration in the Italian road network (Zelaschi et al., 2016).

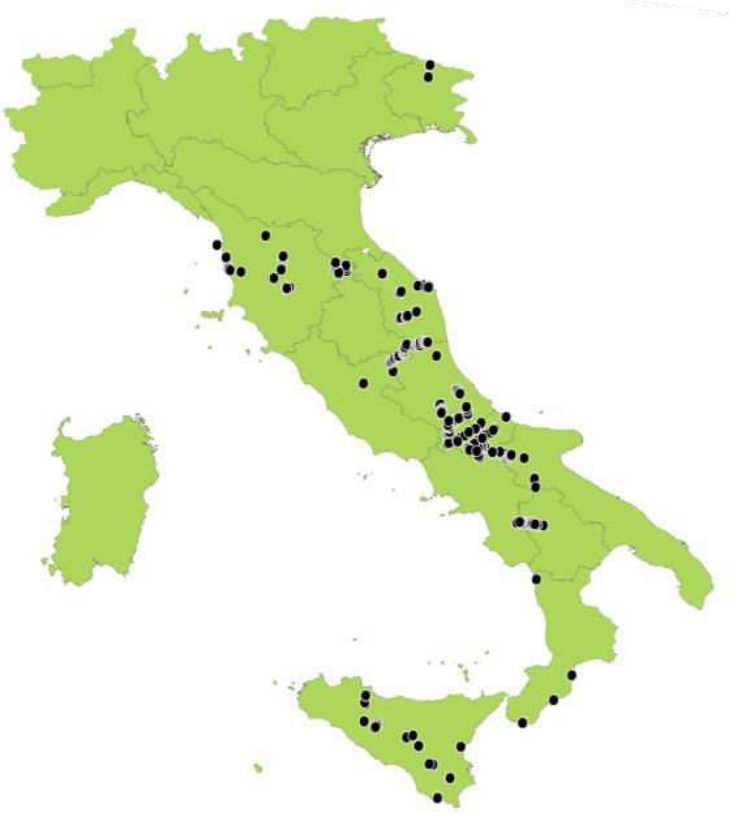

Figure 2: Location of 308 assets in the ANAS bridge inventory used to define the case study

In terms of general dimensions, the overall number of spans ranges from 2 to 36 , corresponding to a length range of $50 \mathrm{~m}$ to $1250 \mathrm{~m}$. A large portion of the inventory bridges are not straight, as $35 \%$ of the assets have curved decks on at least one of the spans. The height of piers ranges between $5 \mathrm{~m}$ and $45 \mathrm{~m}$ in the overall inventory and it is typical to observe large variation of the pier height within the same asset, leading to irregular dynamic configurations within straight bridges. The distributions of these structural configuration properties are shown in Figure 3. In terms of static configuration, the vast majority of the case-study assets have spans that are simply supported upon the piers with thin elastomeric pads, and only a small percentage has continuous deck and bearings that can be either elastomeric or isolators. 


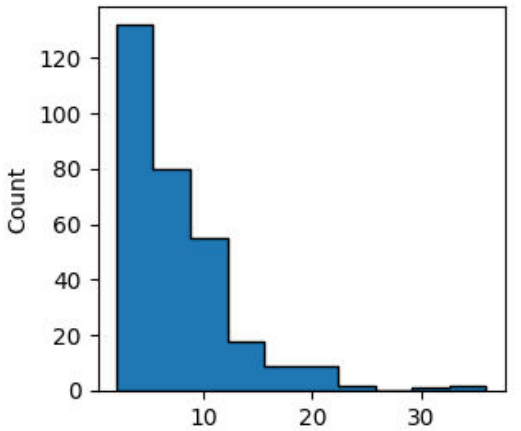

a) Number of Spans

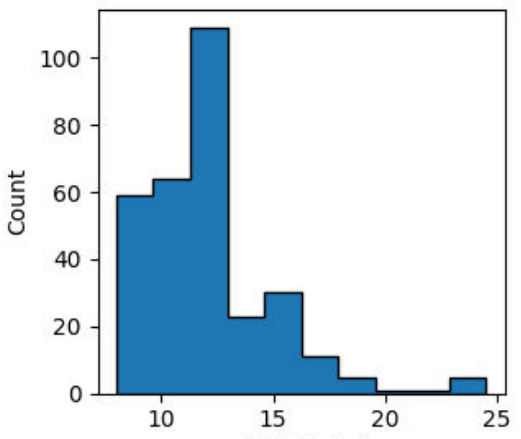

d) Width $(\mathrm{m})$

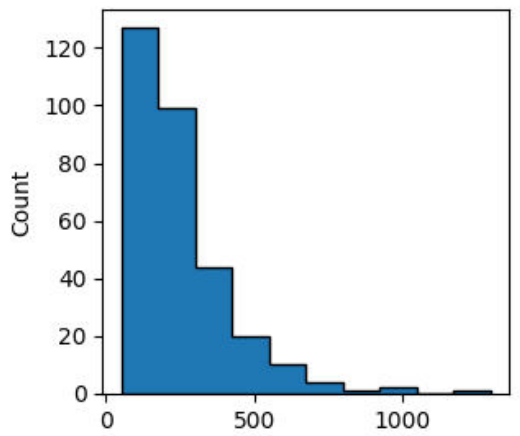

b) Total Length ( $\mathrm{m}$ )

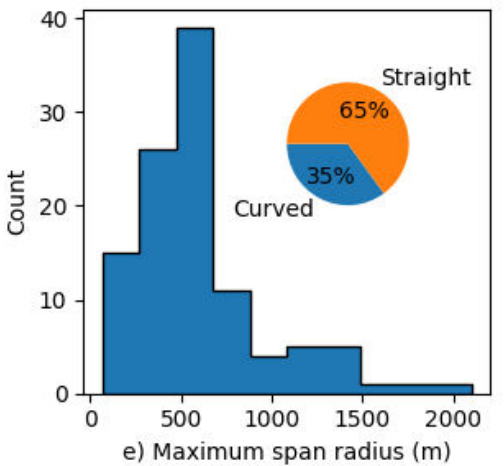

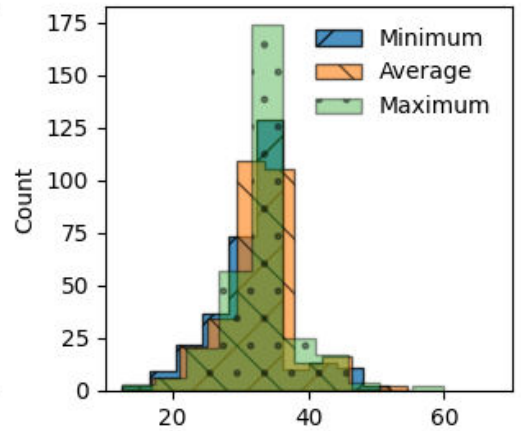

c) Span Length $(\mathrm{m})$

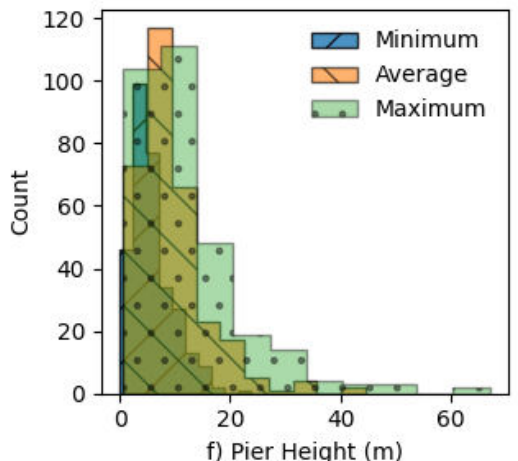

Figure 3: Distribution of general and geometrical properties of the bridge database

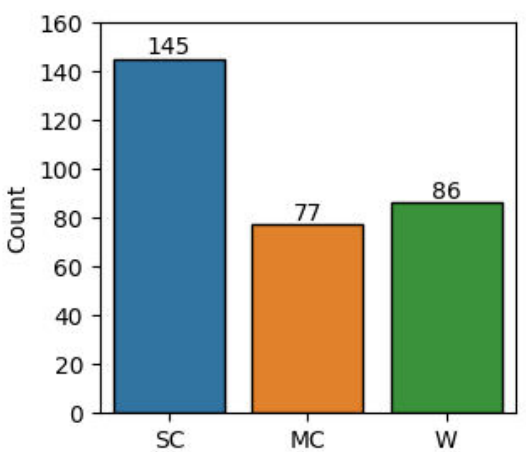

(a) Pier Type

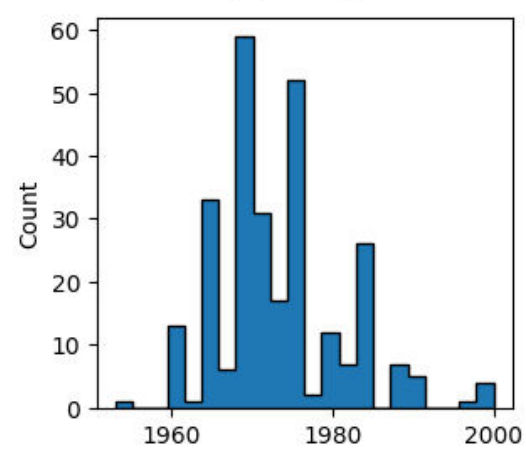

(d) Construction Year

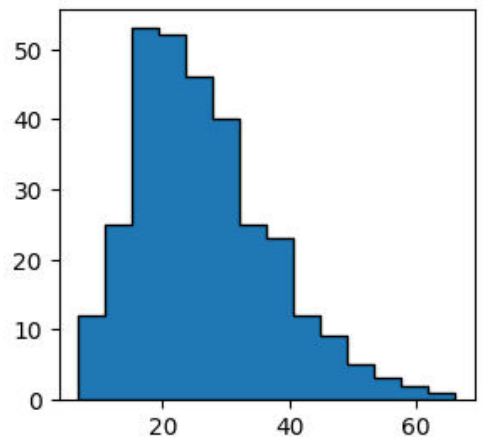

(b) Concrete Strength ( $\mathrm{MPa}$ )

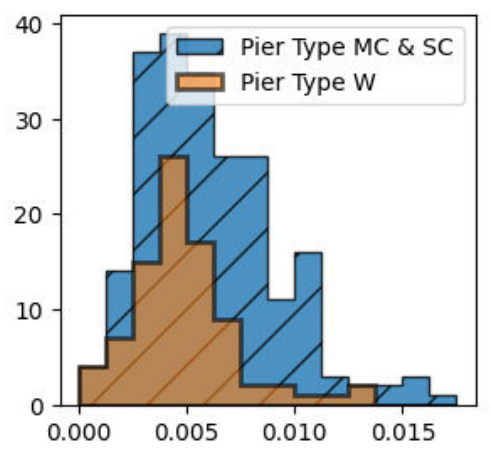

(e) Pier longitudinal steel ratio $\left(A_{s l} / A_{C}\right)$

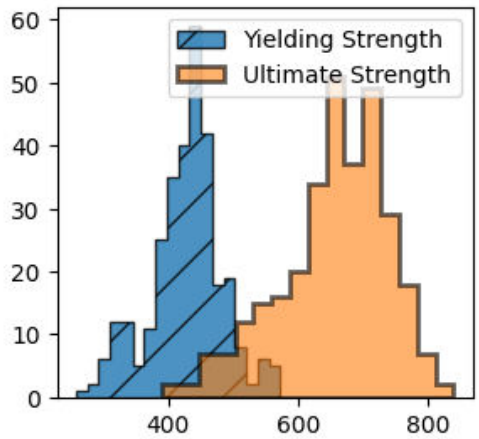

(c) Steel Strength (MPa)

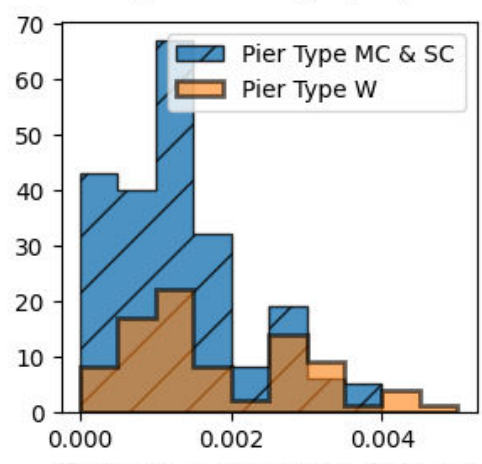

(f) Pier transverse steel ratio $\left(A_{s t} / A_{c}\right)$

Figure 4: Distribution of main material properties of the bridge database (SC: Single Column, MC: Multiple Columns, W: Wall, $\mathrm{A}_{\mathrm{sl}}$ : Area of longitudinal steel, $\mathrm{A}_{\mathrm{st}}$ : Area of transverse steel, $\mathrm{A}_{\mathrm{c}}$ : gross area of the element) 
In terms of pier sections, the inventory includes multiple configurations, which sometimes change even within the same asset. For simplicity in classification, three main pier types were adopted: single column (SC), wall (W) and multiple column (MC) configurations, the distribution of which is shown in Figure 4(a). It is important to note that the actual pier cross sections might be composed of circular sections, box sections, elliptical or many other kinds of geometrical configurations, however, it was opted to aggregate some of these into the pier types to avoid excessive subcategorising and having some pier categories with very few assets to analyze. The construction year was available for all assets, ranging between 1953 and 2000, and most of them were built during the 1960s and 1970s (Figure 4(d)). None of them seemed to have had a design governed by seismic requirements, which was expected, especially considering that the first national seismic regulation in Italy that provided specific design requirements for the entire territory was introduced in 2003 (Consiglio dei Ministri , 2003).

In general, the reinforcement percentages in the piers, both in longitudinal $\left(\mathrm{A}_{\mathrm{sl}} / \mathrm{A}_{c}\right)$ and transverse directions $\left(\mathrm{A}_{\mathrm{st}} / \mathrm{A}_{\mathrm{c}}\right)$, are low in comparison to current design standards and are quite similar across the different pier sections. This is atypical under current design practices, however, both the reinforcement ratios and the properties of the materials used for construction are in line with the age of construction of the inventory. Distributions for the mechanical properties of the materials are shown in Figure 4(e) and (f). In terms of dynamic properties, a structural model was created for each asset to determine the modal periods in both orthogonal horizontal directions. Given that, for the case of bridges, the first mode many times does not account for a significative percentage of the total modal mass (O'Reilly, 2021), an appropriate number of modes were evaluated for each asset to include $85 \%$ of the modal mass in each direction. The distributions for the first modal period $\left(\mathrm{T}_{1}\right)$ and the modal period at which $85 \%$ of the modal mass is obtained $\left(\mathrm{T}_{85 \%}\right)$ are shown in Figure 5.

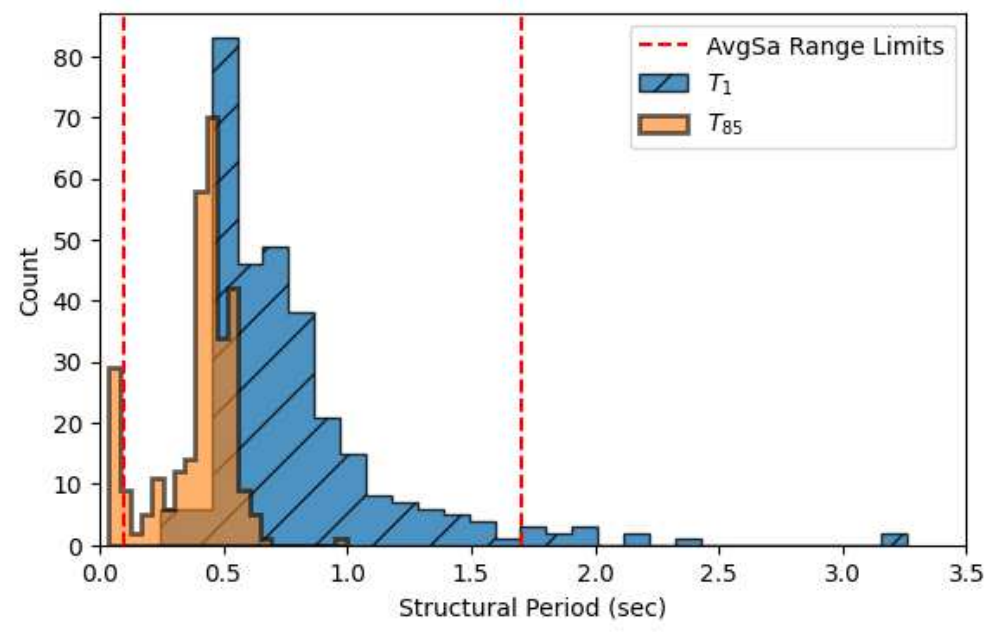

Figure 5: Results for modal structural periods of the entire inventory and definition of AvgSa range

The chosen IM to perform hazard and fragility calculations was the average spectral acceleration (AvgSa), which has been shown to perform well for the risk assessment of bridge portfolios (Abarca et al., 2021). The collective results of $\mathrm{T}_{1}$ and $\mathrm{T}_{85 \%}$ were thus used to define the AvgSa period range. As shown in Figure 5, the selected range was 0.1 seconds to 1.7 seconds, which was defined as per O'Reilly (2021) as 1.5 times the $84^{\text {th }}$ percentile to account for period elongation of the first mode and 0.5 times the $16^{\text {th }}$ percentile to account for higher mode contributions of the $\mathrm{T}_{1}$ and $\mathrm{T}_{85 \%}$ periods, respectively, for the entire inventory. Based on the differences in the number of spans and pier types, nine bridge taxonomy branches, listed in Table 1, were defined. The locations and number of assets based on their respective taxonomy branch are shown in Figure 6. 
Table 1: Definition of taxonomy branches based on key structural parameters

\begin{tabular}{|c|c|c|c|}
\hline Material & Spans & Pier Type & Taxonomy Branch \\
\hline \multirow{4}{*}{$\begin{array}{c}\text { Reinforced } \\
\text { Concrete }\end{array}$} & \multirow{3}{*}{2 to 4 to 8} & Single Column & SC-2to4 \\
\cline { 3 - 4 } & & Multiple Column & MC-2to4 \\
\cline { 3 - 4 } & & Wall & W-2to4 \\
\cline { 3 - 4 } & \multirow{3}{*}{9 to 36} & Multiple Column & SC-5to8 \\
\cline { 3 - 4 } & & Wall & MC-5to8 \\
\cline { 3 - 4 } & & Multiple Column & W-5to8 \\
\cline { 3 - 4 } & & Wall & MC-9to36 \\
\hline
\end{tabular}

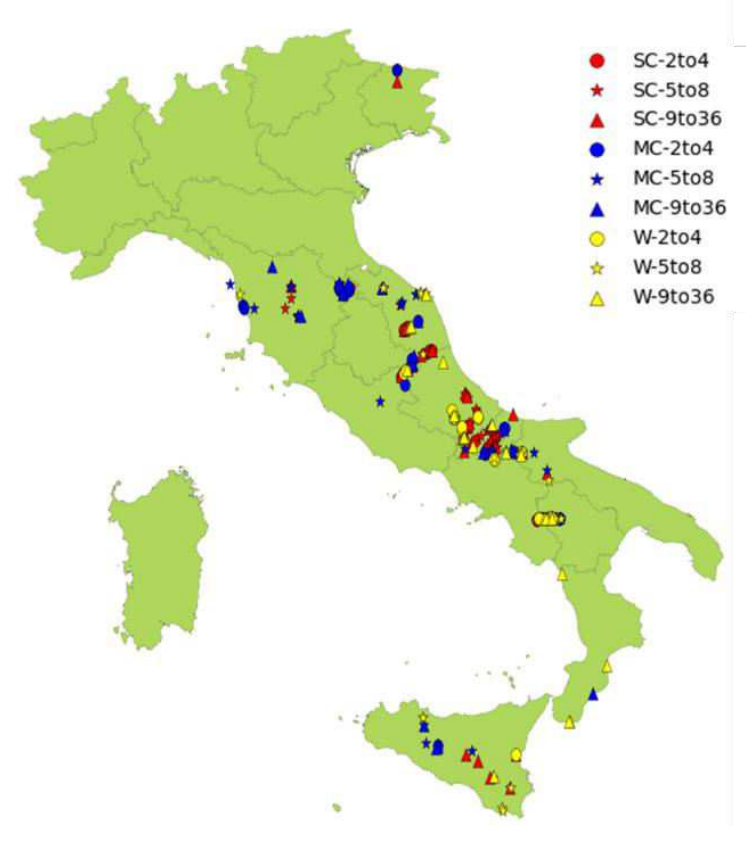

(a)

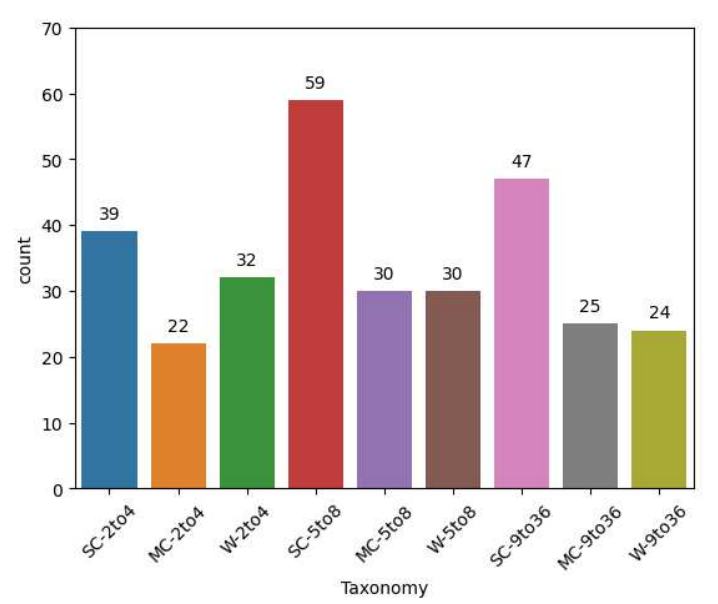

(b)

Figure 6: Distribution of taxonomy branches in database: (a) Location of assets based on taxonomy branch, (b) Number of assets per taxonomy branch present in the database

\subsection{Case study sampling}

As shown in Figure 2, the bridges in the ANAS database are scattered geographically over the Italian territory and not directly connected. Therefore, they are not an ideally positioned to define an illustrative case study, if using their current locations. Moreover, even in locations with different seismic hazard demands, bridge design practice does not vary considerably among the Italian territory for the construction period of the bridges in the database (Borzi et al., 2015). For these reasons, a synthetic case study was created by taking the road network of a region for which the location of bridges is known and assigning assets from the database in each of those locations. 
In this sense, the Salerno region was selected for having a relatively varied seismic hazard demand and a somewhat lower number of bridges, thus reducing the need to resample the same bridge from the database on the case-study region a large number of times. Information about the road network of Salerno was taken from the OpenStreetMap database (OpenStreetMap contributors, 2020), which comprises all roads within the highway, primary and secondary systems, including a total of 617 bridges. The 308 bridges in the database were thus located randomly within the locations of bridges in the Salerno network using a sampling with replacement scheme. In order to generalize the case study and strengthen the results of the current research, ten different case studies were created by repeating the sampling procedure, hence obtaining ten different configurations of asset types in the locations of bridges in the Salerno network. In order to maintain the validity of the fragility results and avoid having to run NLTHA with different earthquake record sets for the same bridges, the sampling process was carried out considering fixed combinations of seismic zones and soil types for each bridge. Examples of two case-study realisations are shown in Figure 7 with respect to the resulting spatial distribution of the taxonomy branches and the number of occurrences of each branch in the realisation.

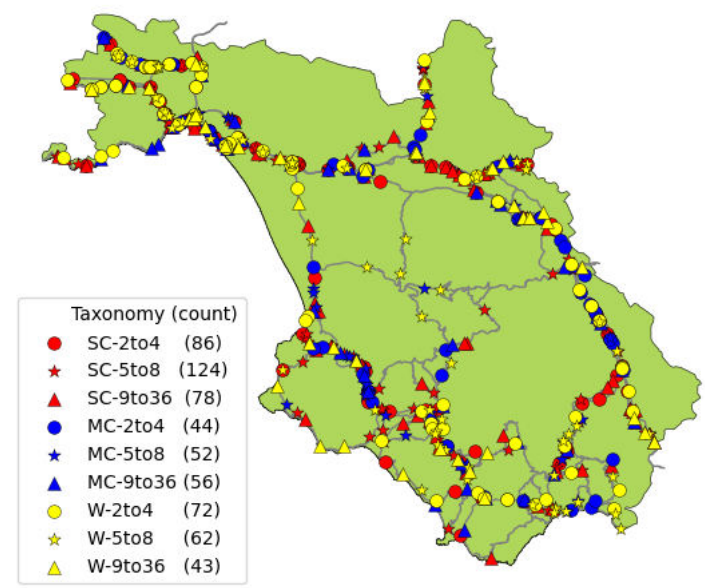

(a)

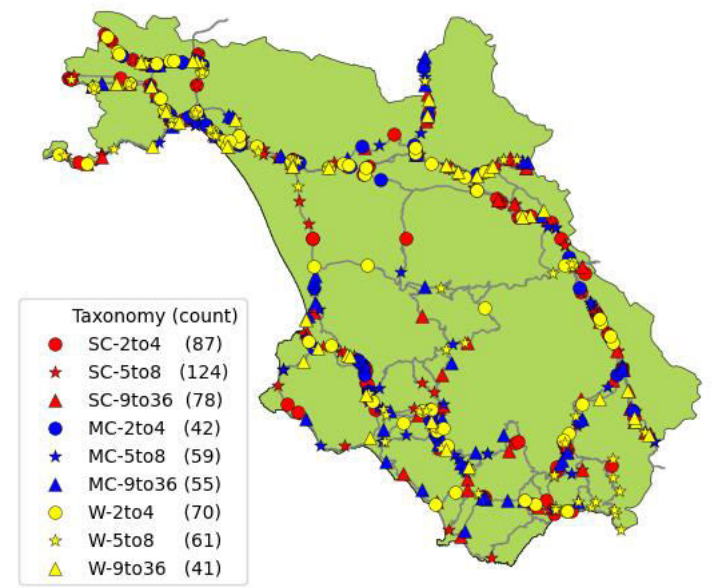

(b)

Figure 7: Examples of sampling used to define Case Study realisations: (a) Case Study number 1, (b) Case Study number 3

\section{Hazard: PSHA and ground motion selection}

The case-study region has a varied seismic hazard that ranges from low seismicity regions near the coastline, to high seismicity areas near the Southern Apennines Mountain range. This wide range of seismicity represents an opportunity for this study since it allows for possible differences in the response of bridges in different seismic demand areas to be accounted for.

In terms of hazard curves, the SHARE hazard model (Woessner et al., 2015), implemented in the OpenQuake Engine (Silva et al., 2014), was used to determine the probability of exceedance, in an investigation period of 50 years at each bridge site, of the different AvgSa levels in the period range of $0.1 \mathrm{~s}-1.7 \mathrm{~s}$. In terms of ground motion record selection, a conditional spectrum scheme (Lin et al., 2013) was adopted using a modification that allows the conditioning of the spectra for AvgSa (Kohrangi et al., 2017) was followed using the EzGM tool developed by Ozsarac et al. (Ozsarac et al., 2021).

The implementation of the adopted record selection methodology requires results from a disaggregation analysis to determine the mean magnitude and distance that drive the seismic demands for the adopted IM 
1 at each specific site. However, given the large number of bridge locations and to minimize the 2 computational burden of performing disaggregation at each location, all assets were assigned to four 3 hazard zones and two soil classes (soft and stiff soil differentiated by $\mathrm{V}_{\mathrm{s} 30}$ ) as shown in Figure 8, after 4 which a complete hazard disaggregation analysis was carried out for the eight possible zone-soil 5 combinations as shown in Figure 9(a). For each combination, sets of 30 bidirectional earthquake records 6 were selected from the NGA West-2 Strong-motion Database (Bozorgnia et al., 2014) for nine return 7 periods ranging from 98 years to 9975 years, to be used for nonlinear time-history analysis (NLTHA), as 8 described in Section 5. An example set of the records obtained is shown in Figure 9(b).

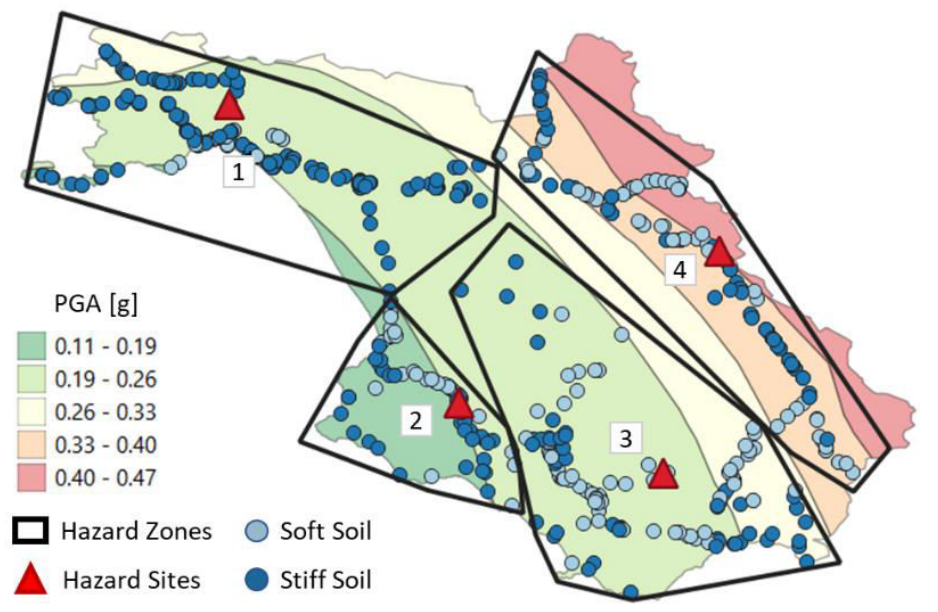

(a)

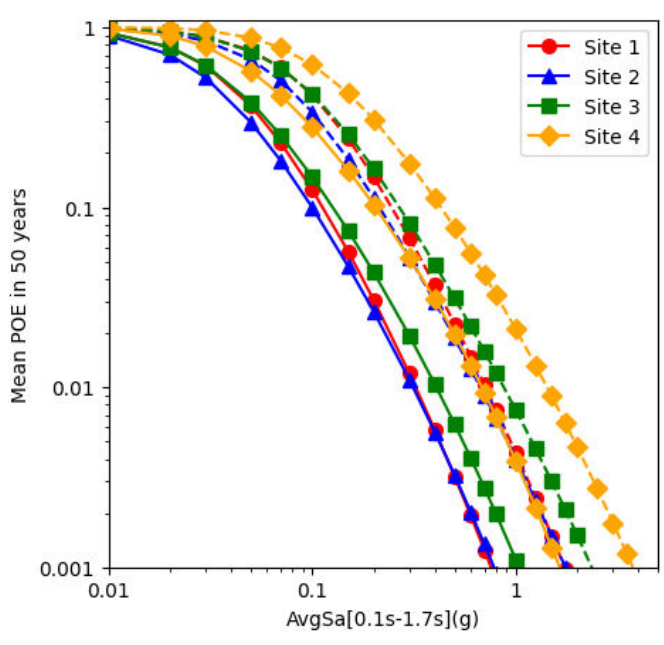

(b)

Figure 8: Seismic hazard of the case study region of Salerno: (a) Hazard zones and soil sites (PGA values for a return period of 475 years are shown for reference), (b) Hazard curves for each hazard zones (dashed lines are soft soil results) 

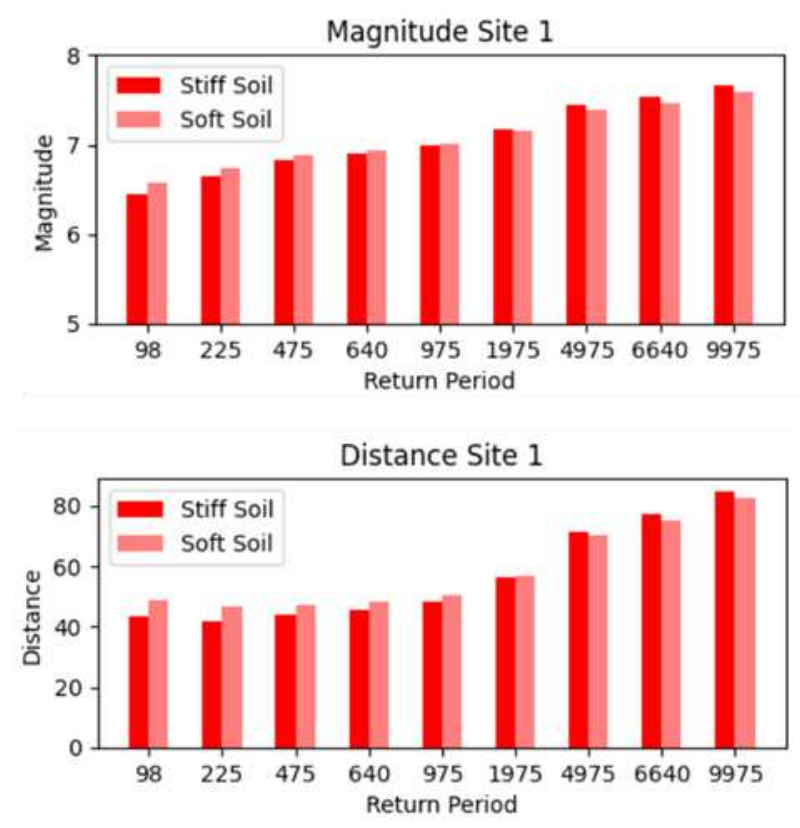

(a)

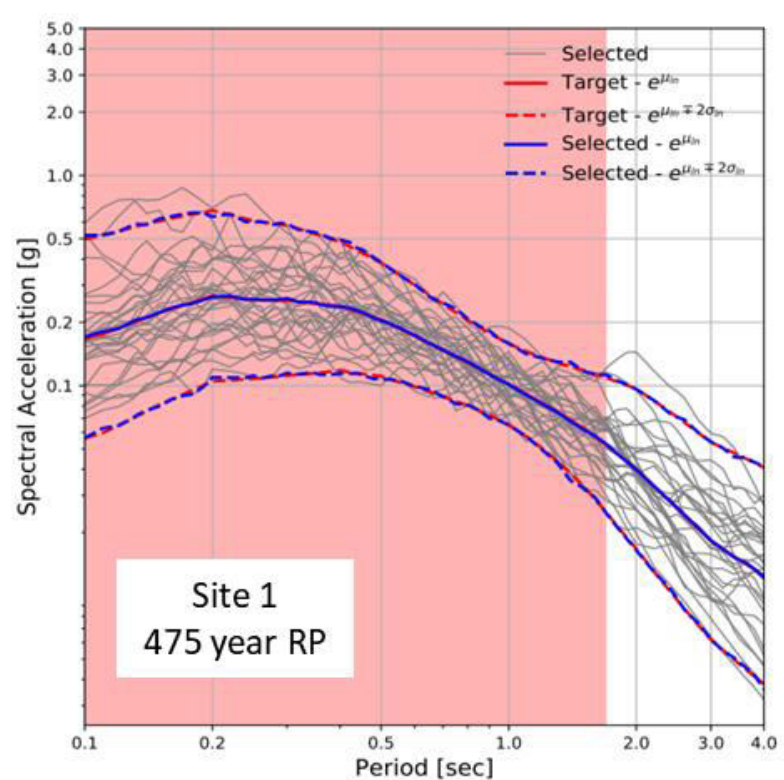

(b)

Figure 9: Conditional Spectrum Record Selection: (a) Disaggregation results for Site 1, (b) Example of record selection for Site 1, 475-year return period, stiff soil

\section{5. Vulnerability: fragility and direct loss assessment}

Taking advantage of the complete knowledge of the structural characteristics of all the elements in the database, the BRI.T.N.E.Y tool (Borzi et al., 2015) was adopted to evaluate the fragility of each bridge in the portfolio to seismic events. Since the focus of this study was not the derivation of fragility curves for bridges, the specificities of the numerical models created and the overall analysis procedure will not be explained in detail herein hence interested readers are encouraged to refer to the publication by Borzi et al. (2015). Overall, the methodology relies on NLTHA of numerical models created for each asset. It used an automated set of codes to record the performance of the structure for each earthquake record selected as a sample of demand over capacity ratios. This is then used to fit a lognormal distribution of performance for each intensity measure as shown in Figure 10. These distributions are used to evaluate the exceedance of specific limit states and fit a lognormal fragility curve for each bridge. In this study, the fragility analysis was focused on the evaluation of the collapse limit state, defined as the exceedance of the bridge capacity in terms of flexural rotation capacity, shear failure or unseating of the deck in both the longitudinal and transverse directions. 


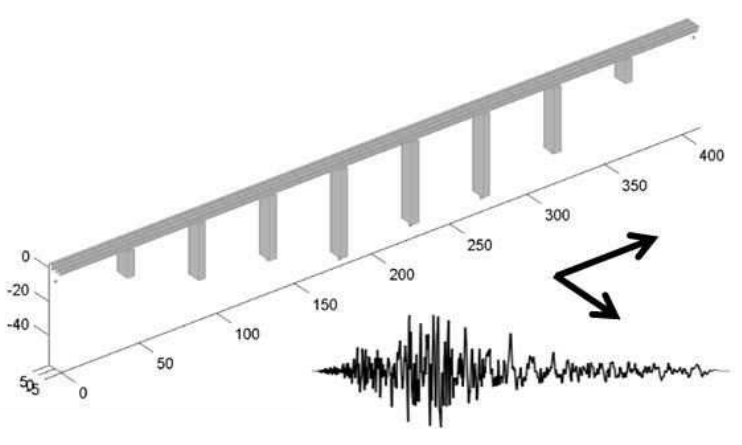

(a)

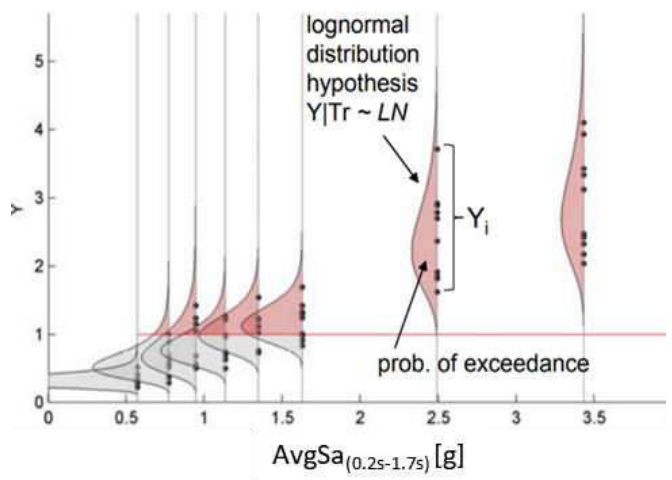

(b)

Figure 10: Fragility assessment using BRI.T.N.E.Y: (a) Numerical model created with BRITNEY subjected to bi-directional ground motion, (b) Determination of probability of exceedance per return period (adapted from Borzi et al., 2015)

The results obtained for the fragility curves of each element in the inventory, separated by taxonomy branch, are shown in Figure 11, where the mean fragility curve is shown for reference. This lognormal mean fragility curve is represented by the average of all the mean fragility curves of the different bridge models, as per Equation 1. The overall dispersion is given by the square root of the sum of squares of the intra-bridge dispersion and the inter-bridge dispersion, as presented in Equations 2 to 4.

$$
\begin{array}{r}
\ln \mu_{\ln Y_{t a x}}=\frac{1}{N} \sum_{i=1}^{N} \ln \mu_{\ln Y_{i}} \\
\beta_{\ln Y_{\text {tax }}}=\sqrt{\beta_{\ln Y_{\text {intra }}}^{2}+\beta_{\ln Y_{\text {inter }}}^{2}}
\end{array}
$$

where:

$$
\begin{aligned}
& \beta_{\operatorname{lnY} Y_{\text {intra }}}=\frac{1}{N} \sum_{i=1}^{N} \beta_{\ln Y_{i}} \\
& \beta_{\text {lnY } Y_{\text {inter }}}=\sqrt{\frac{\sum_{i=1}^{N}\left(\ln \mu_{\ln Y_{i}}-\ln \mu_{\ln Y_{\text {tax }}}\right)^{2}}{N}}
\end{aligned}
$$



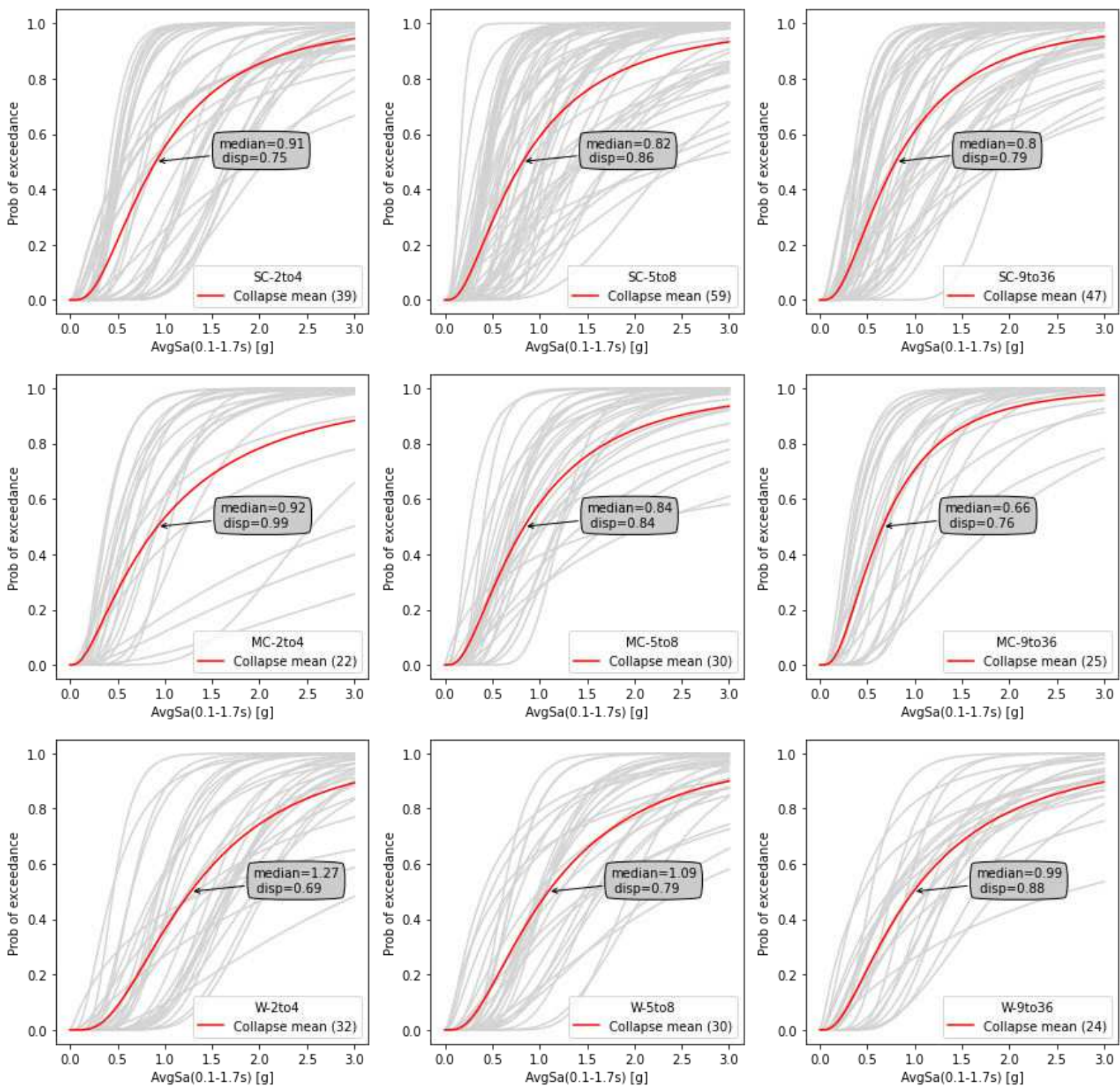

Figure 11: Fragility curves for Collapse Limit State obtained for the 308 bridges in the database separated by taxonomy branch

The calculation of AALs associated with the collapse limit state was carried out using the formulation from the Pacific Earthquake Engineering Research Center's Performance-Based Earthquake Engineering (PEER PBEE) framework (Porter, 2003). A very straightforward implementation of the formulation is possible by including only the collapse limit state, where the product of the annual probability of exceedance of the limit state times the direct replacement cost will result in the direct collapse-based AAL, as described by Equation 5.

$$
\mathrm{AAL}=p\left(L S_{C}\right) \cdot € L \mid L S_{C}=A P E_{C} \cdot € R C
$$

where:

$L S_{C}$ : Collapse Limit State $p\left(L S_{C}\right)$ : probability of occurrence of $L S_{C}$
$A P E, L S_{C}$ : annual probability of exceedance of $L S_{C}$ $€ R C$ : bridge replacement cost 
$€ L \mid L S_{C}$ : direct economic losses associated to $L S_{C}$

1 The annual probability of exceedance (APE) for the limit state was obtained by convoluting the fragility 2 and hazard curves obtained for each bridge in each case study. The replacement cost for each bridge was 3 taken as proportional to the deck area, considering a generic cost per square meter of €930, taken from the 4 mean replacement cost per area assumed by Perdomo et al. (2020) for a similar Italian bridge inventory. 5 The results for collapse-based direct AAL are show in Figure 12 for the two example case studies previously presented in Figure 7, whereas Table 2 summarizes the total AAL calculated for the entire portfolio configuration in each case-study realization. These aggregated loss values per case study will be used as a benchmark to evaluate the uncertainty related to the exposure knowledge level in the following sections.

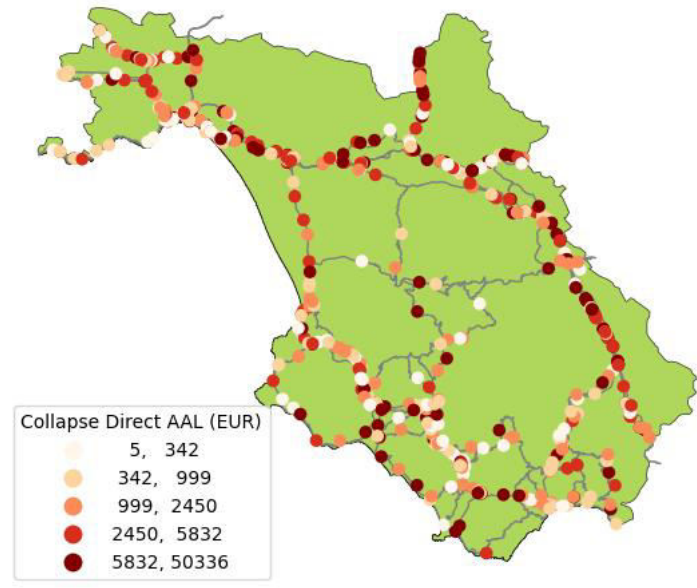

(a)

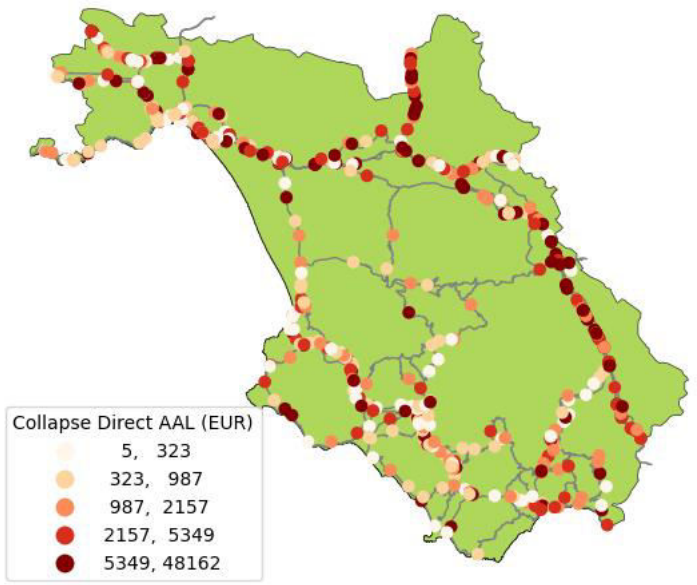

(b)

Figure 12: Results for collapse AAL: (a) Case Study number 1, (b) Case Study number 3

11

Table 2: Summary of baseline total portfolio direct loss per case study

\begin{tabular}{|c|c|}
\hline Case Study & Total Direct Loss \\
\hline 1 & $€ 2,552,567$ \\
\hline 2 & $€ 2,240,684$ \\
\hline 3 & $€ 2,291,323$ \\
\hline 4 & $€ 2,525,752$ \\
\hline 5 & $€ 2,296,542$ \\
\hline 6 & $€ 2,338,223$ \\
\hline 7 & $€ 2,332,061$ \\
\hline 8 & $€ 2,314,442$ \\
\hline 9 & $€ 2,211,758$ \\
\hline 10 & $€ 2,277,621$ \\
\hline
\end{tabular}




\section{Evaluation of exposure uncertainty}

\subsection{Taxonomy-based approach}

The use of taxonomy-based curves is rooted in the assumption that assets with similar configurations will have a similar performance or damage when subjected to equal levels of seismic demand. Therefore, macro fragility curves created for classes of structures can be used for assets within the class without detailed analysis. As shown schematically in Figure 13, if all the assets in a case study were of the same taxonomy branch, specific analysis could be carried out on the bridges for which complete structural information is available. This then allows the individual fragility curves for each of them to be obtained. Subsequently, a mean fragility curve can be assembled by accounting for the mean responses, along with the inter and intra dispersion of the curves, given by Equations 1 to 4 . This would constitute the taxonomy-based fragility curve for the class that can be used for all the remaining assets in the taxonomy branch that have incomplete information.

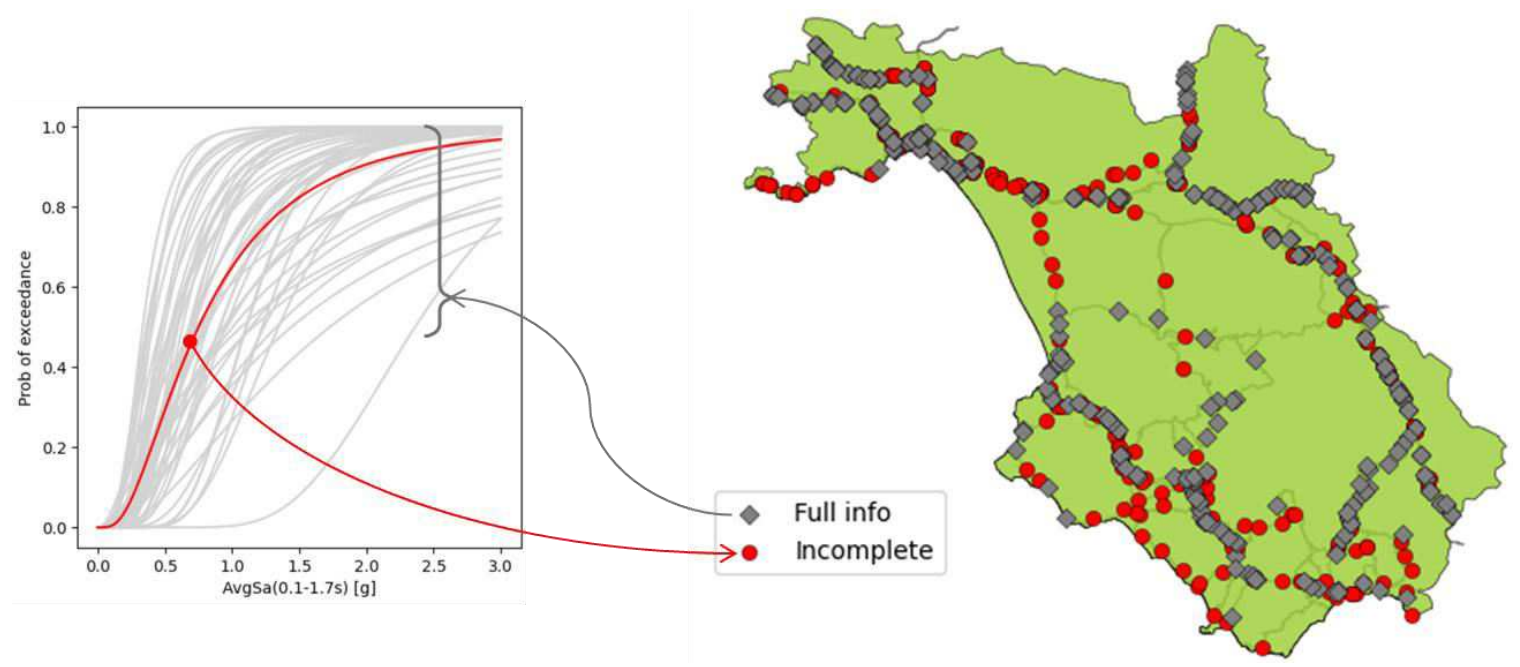

Figure 13: Schematic representation of taxonomy-based fragility curve assignment on assets of the same taxonomy branch with incomplete information

While this assumption is generally accepted for regional-level seismic risk assessments, it is expected that such a simplification will introduce a non-negligible level of uncertainty, depending on the classification scheme that is used to define the branches and the number of bridges per branch with complete information specifically analysed. Since there is no consensus on a definitive classification system, or on the number of bridges required to be analysed to properly characterise a taxonomy branch, risk analysts will typically make these decisions based on the information that is available.

In order to have a comprehensive evaluation of the inherent accuracy of the use of taxonomy-based approaches, the classification system introduced in Table 1 is used to assign a taxonomy branch to each asset in the case-study portfolio realisations described in Section 3.2. Once this is done and a baseline collapse-based direct AAL estimate for each case study is performed, increasing portions of the portfolio's results are randomly removed. The remaining values are used to calculate taxonomy-based fragility curves that are then assigned to each asset of the case studies with removed information based on their classification. The ratio of known over unknown portions of the inventories explored in this study, hereinafter referred to as exposure rates, ranges from $5 \%$ to $100 \%$ in $5 \%$ increments, leading to 20 different exposure rates. 
Additionally, the taxonomy-based curves calculated for each exposure rate will change depending on the specific assets available in the known portion of the portfolio. This is illustrated in the example shown in Figure 14, where four different samples of the same exposure rate for the assets in a taxonomy branch from a case-study realisation will yield slightly different mean curves. To account for this, 40 different random samples of assets with full information are taken for each exposure rate.
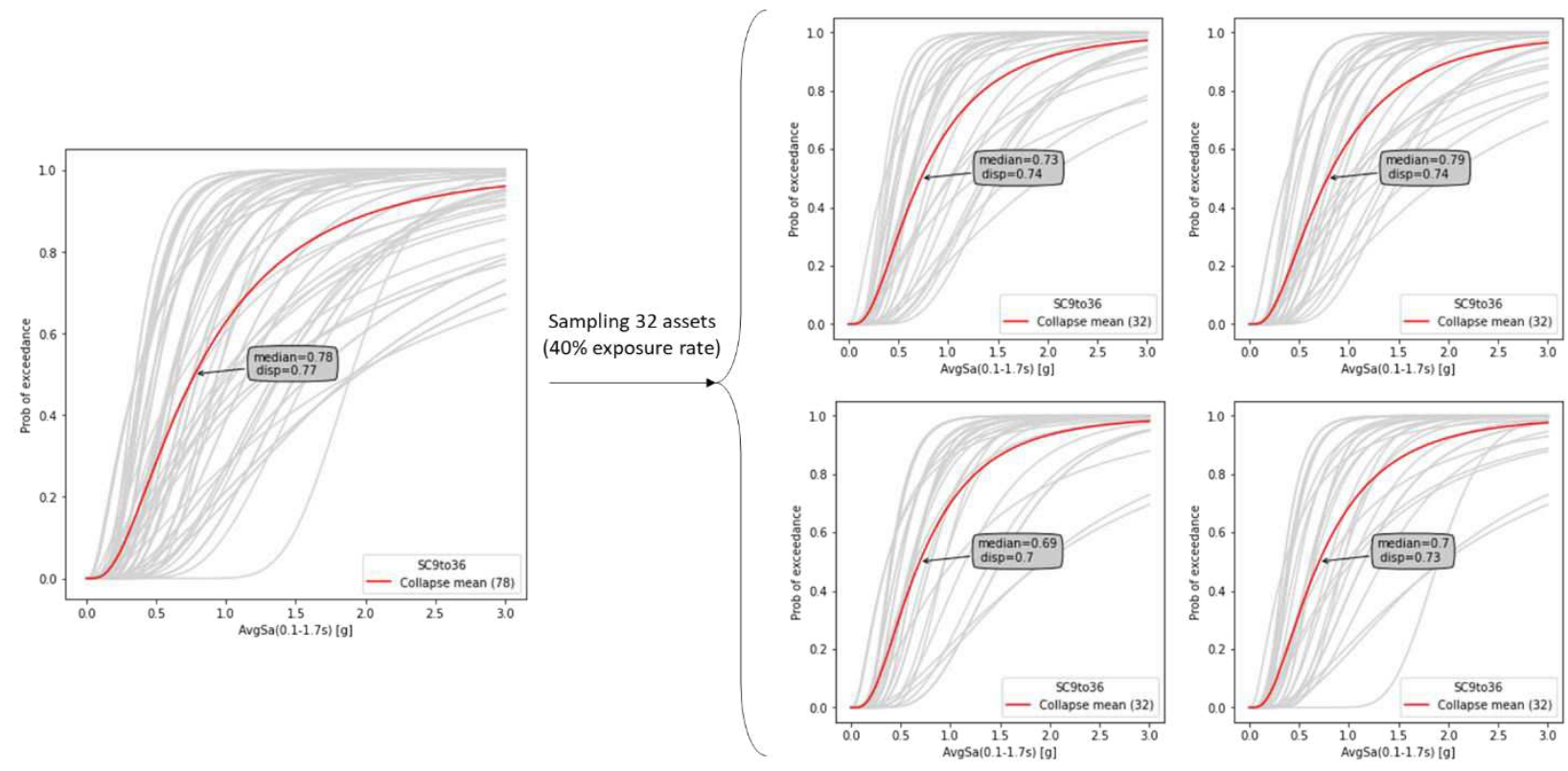

Figure 14: Example of variation in taxonomy-based curves obtained by sampling a $40 \%$ exposure rate from the RC-SC-9to36 taxonomy branch for Case Study 1

For each combination of the 10 case study realizations, 20 exposure rates and 40 known asset samples (8000 iterations in total), a recalculation of losses was carried out using the specific curves determined for the known portions of the assets. The taxonomy-based curves were then applied to the unknown assets, leading to a new estimate of the total direct AAL for the entire portfolio that can be compared to the baseline calculation for each case study realization. Results obtained for the inaccuracy in calculation with each exposure rate evaluated are shown in Figure 15. It can be seen that, as expected, the uncertainty in the calculation of total direct collapse AAL for the entire portfolio reduces as the proportion of assets with full information increases. Furthermore, the uncertainty associated with \pm 2 standard deviations has a highly nonlinear trends up to an exposure rate of about $30 \%$, after which the reduction becomes almost linear. As such, the $30 \%$ threshold seems to be a good threshold for the minimum amount of assets within a portfolio that should be analysed when using taxonomy-based fragility curves, since obtaining more complete exposure information above this point leads to a much lower increase in accuracy in the overall results. In addition to the uncertainty estimates, median values of prediction, calculated using taxonomybased curves, are consistently close to the baseline results calculated with individual curves specific to each asset. This is in line with findings made in previous studies (Abarca et al., 2021) and further demonstrates that the use of taxonomy-based curves can lead to accurate mean estimates of the total direct losses for the entire portfolio. 


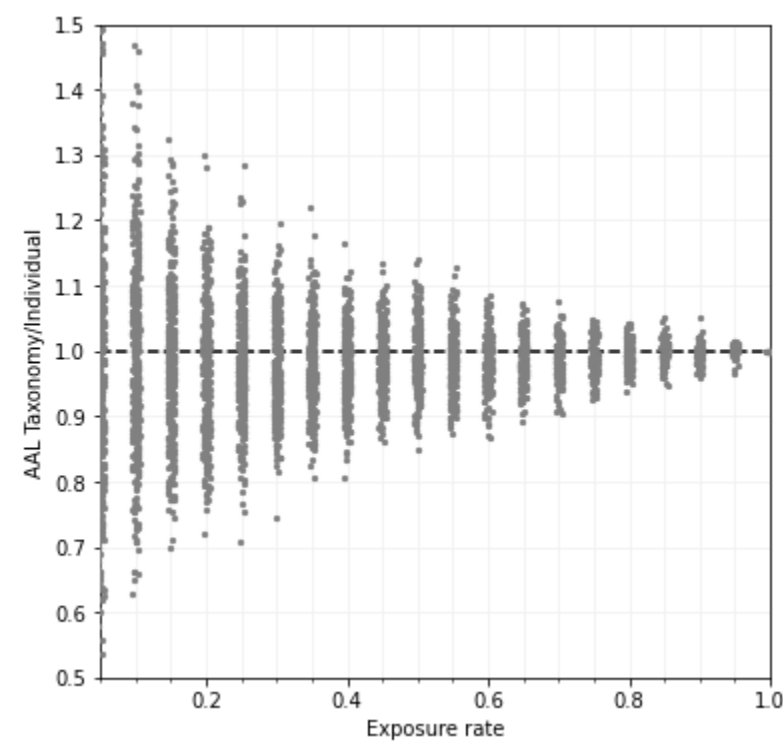

(a)

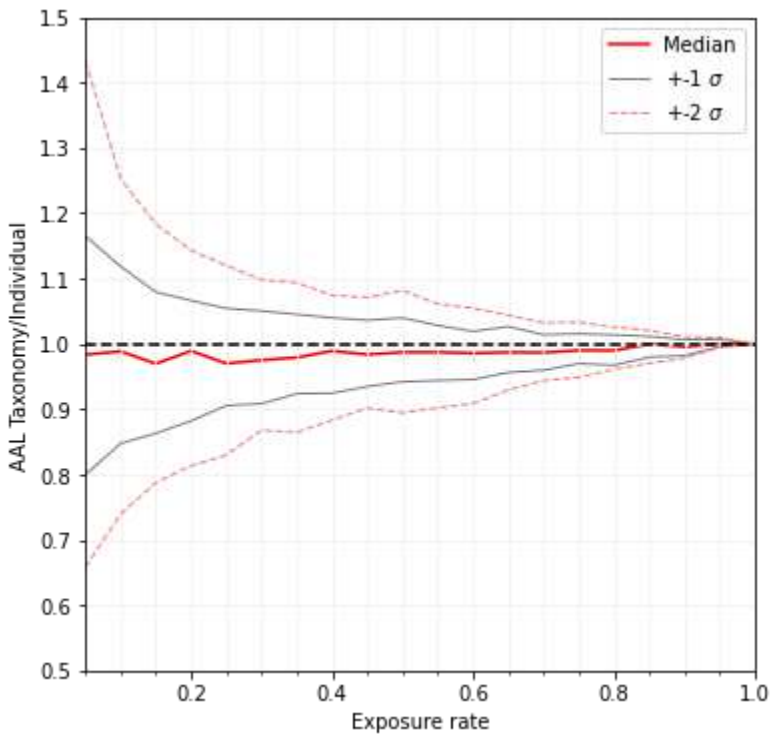

(b)

Figure 15: Results of total inventory direct AAL Taxonomy over Individual based on different exposure knowledge percentages: a) results for each iteration, b) statistical trends observed in results

\subsection{Machine learning model}

A supervised machine learning model was evaluated in terms of its capacity to reduce the uncertainty in calculations deriving from the lack of exposure data to assess individual structures in a portfolio. The objective of this evaluation was to predict the fragility curves of assets with unknown information within the same sets of taxonomy branches by using simple structural geometrical parameters. These parameters differentiate each asset within the class and are used to predict a suitable fragility curve for each bridge, using the results from the portion of the inventory with full information. This contrasts with the taxonomy-based approach that uses the same mean fragility curve for all the unknown assets within the taxonomy branch, regardless of the variations between geometric characteristics of elements within the same class that could also influence their structural performance.

For this purpose, a machine learning model is built for each taxonomy branch and trained using the database of NLTHA results of assets with full information to predict the probability of exceeding the collapse limit state given simple bridge geometrical parameters and an IM level. These models are then used for each bridge with incomplete information to predict their probability of exceedance of the limit state at discrete points of IML, after which a continuous fragility curve is fitted and assigned to each corresponding bridge.

A Random Forest Classification Model was chosen given its good performance when compared to other machine learning algorithms for similar endeavours recently demonstrated by (Mangalathu et al., 2019). This type of algorithm uses a collection of decision trees built with bootstrapped subsets of the NLTHA database. Each tree is fitted to provide predictions of the occurrence of collapse based on its sub-sample and all predictions provided by each tree are later weighted to determine the probability of exceedance of the collapse limit state, as depicted graphically in Figure 16. This type of model, as with most supervised machine learning models, uses a labelled dataset that has both its independent variables (inputs) and its outcomes. Moreover, it progressively calibrates its own numerical properties to produce an inferred function that makes predictions about the output values. 

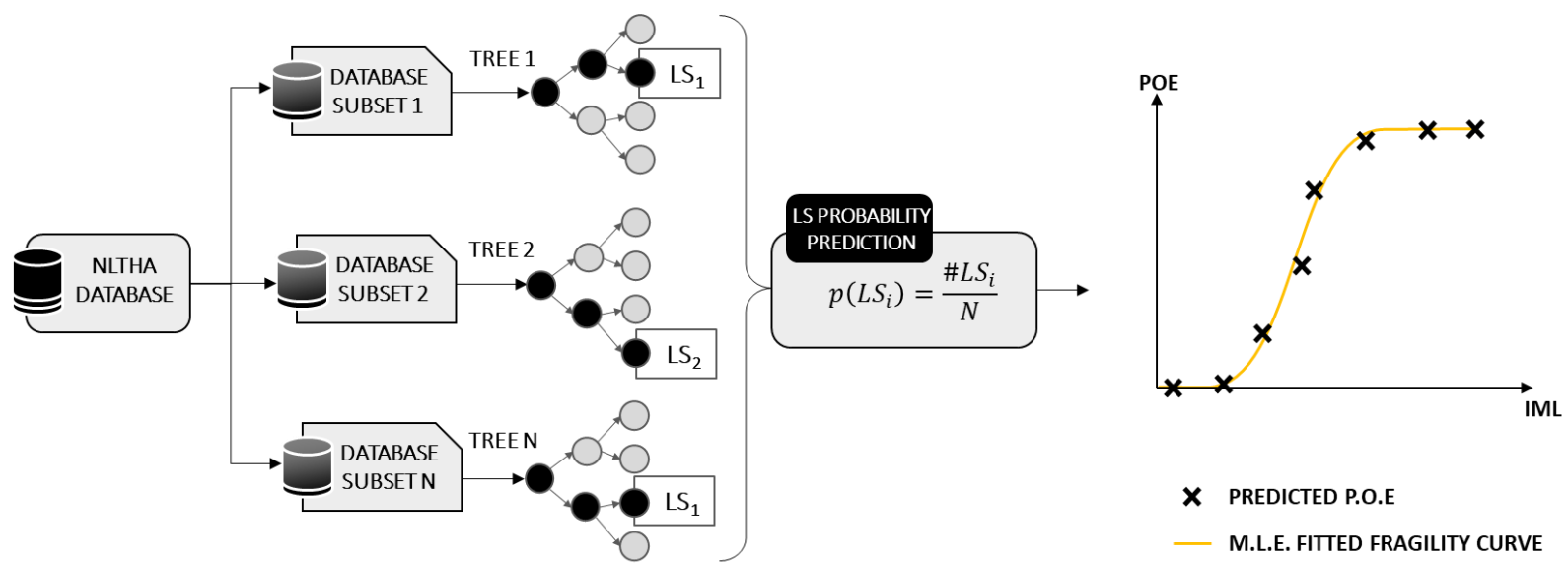

Figure 16: Schematic representation of random forest algorithm prediction methodology

The same combinations between case study realizations, exposure rates and known asset samples used for the taxonomy-based approach were analysed. The NLTHA results of the known portions of the inventories were used to train the random forest models and their prediction results were employed to determine the fragility curves of the assets with incomplete information. Subsequently, an estimate of the total direct AAL for the entire portfolio was computed and compared to the baseline calculation for each case-study realization, as done previously for the taxonomy-based approach.

For each iteration and each model, a database was assembled using the results for each bridge that was sampled as having complete information. The occurrence of collapse as a binary operator (i.e., 1: Collapse, 0: Non-collapse) representing the dependent variable (target) and a vector of independent variables (or features) was retrieved for each ground motion result obtained during the NLTHA process. A set of six features were used: number of spans, total length, average span length, maximum pier height, deck width and the IM level of each ground motion record. Given that these variables, to be processed by the Random Forest algorithm, have different units and orders of magnitude, each was modified using a minimum-maximum scaling process that transforms the data of each feature by scaling the values within the 0 and 1 range.

In terms of the properties assigned to the Random Forest algorithm, a different model is created for each of the nine taxonomy branches at each of the performed 8000 iterations and determining and implementing optimal parameters for each model would be unpractical. As such, the same settings shown in Table 3 were used for all models; these values were determined by averaging the optimised settings for a discrete set of tests performed during a calibration stage.

Table 3: Main parameters selected for the Random Forest Algorithm's implementation

\begin{tabular}{|l|c|}
\hline \multicolumn{1}{|c|}{ Parameter } & Value \\
\hline Number of estimators & 20 \\
\hline Maximum Tree Depth & 12 \\
\hline Maximum Features & $\sqrt{\# \text { features }}$ \\
\hline Minimum Leaf Samples & 2 \\
\hline Minimum Split Samples & 2 \\
\hline
\end{tabular}

The resulting performance of the models changes depending on the exposure rate: the lack of data present when evaluating lower exposure rates leads to a very low accuracy in the prediction of the probability of occurrence of collapse, which reflects on the definition of the fragility curves for the unknown assets. In 
turn, as higher rates are evaluated the performance improves. As an intermediate example, the performance of a single model, evaluated on the assets with incomplete information and created for the RC-SC-9to36 taxonomy branch, using an exposure rate of 50\%, is shown in Table 4 and Figure 17.

Table 4: Example performance of Random Forest model iteration on Case Study 1, Exposure rate $=0.5$, RC-SC-9to36 taxonomy branch

\begin{tabular}{|c|c|c|c|c|c|}
\hline \multicolumn{4}{|c|}{ Classification Confusion Matrix } & \multicolumn{2}{|c|}{ Prediction of Probability of Collapse } \\
\hline & $\mathrm{NC}$ & C & Recall & Root-mean-squared error (RMSD) & 0.151 \\
\hline NC & 4459 & 389 & 0.92 & Mean absolute error (MAE) & 0.078 \\
\hline C & 671 & 1831 & 0.73 & Median absolute error (MedAE) & 0.021 \\
\hline Precision & 0.87 & 0.82 & 0.86 & Coefficient of determination (R2) & 0.829 \\
\hline
\end{tabular}

The confusion matrix for the classification of the intermediate example is presented in Table 4. It consists of a table that records the number of correct and incorrect predictions given by an algorithm, and can be used to evaluate the performance of the model to predict the occurrence of collapse. In this table, the predicted results (organized in columns) are correlated with the actual assignments (organized in rows), while the resulting diagonal elements represent the limit state assignments that were correctly predicted by the model. It can be seen that the accuracy of the model, calculated as the ratio of the assignments that are correctly predicted to the total data, is $86 \%$. This is rather good performance and is in line with similar previous research exercises (Mangalathu et al., 2019). A reduction in performance is observed in terms of the prediction of the probability of occurrence of the collapse limit state, as demonstrated by the metrics provided in Table 4 and Figure 17(a). In fact, it can be seen that, while the typical magnitude of the prediction inaccuracy (described through the mean absolute error) is relatively low at $8 \%$, the performance does not seem to be uniform across the possible range of exceedance probabilities with intermediate values showing larger residuals with a trend towards underprediction.

20 Using the predictions of probability of exceedance to determine the fragility curves of the assets with unknown information in the intermediate example gives the results shown in Figure 17(b). It can be seen that the predicted curves have a similar distribution as the calculated curves for the same bridges, with a slight tendency to underestimate the 'real' fragility of the assets, which is in line with the underprediction of probability of exceedance mentioned previously.
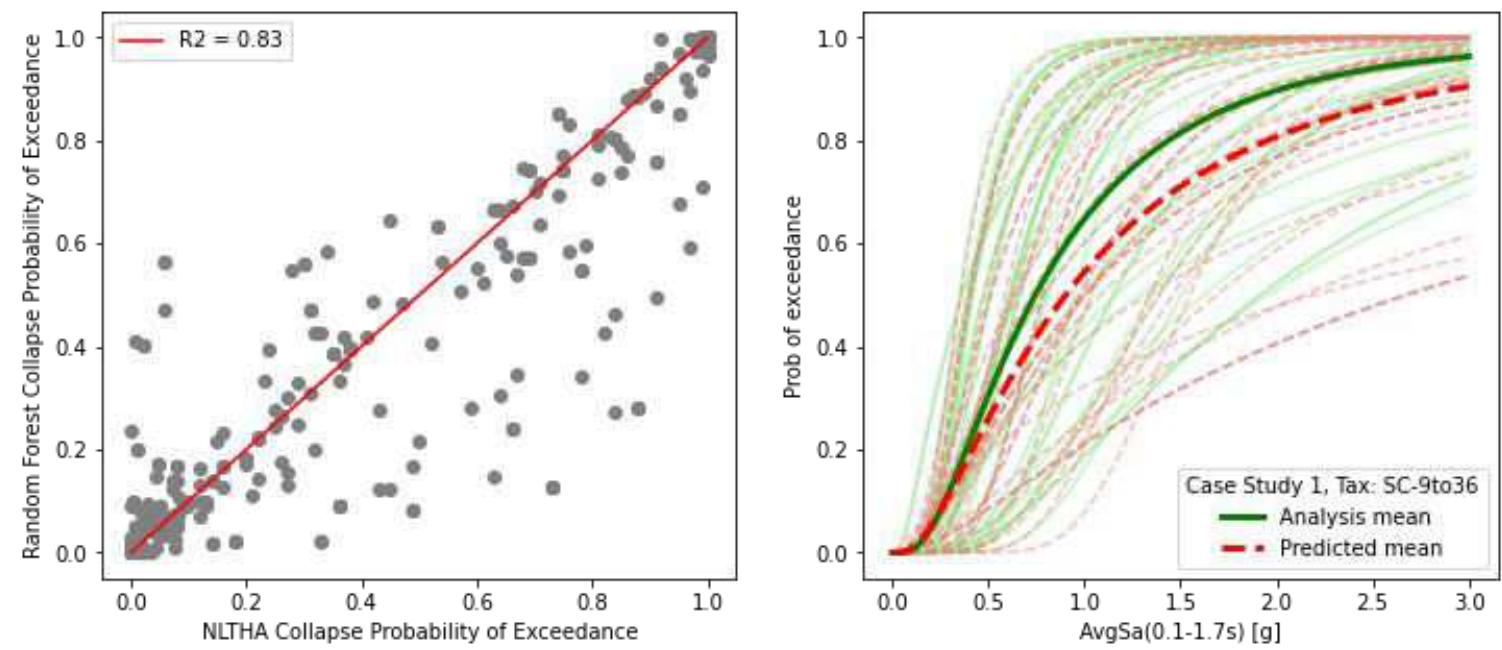
Figure 17: Example performance of Random Forest model iteration on Case Study 1, Exposure rate $=0.5$, RC-SC-9to36 taxonomy branch: a) Prediction of collapse probability of exceedance for unknown bridges,

b) Collapse fragility curves fitted from probability predictions

Overall, the processing of direct collapse AAL for the entire case study over each iteration leads to the results shown in Figure 18. In this case, as with the taxonomy-based approach, the uncertainty in the calculation of total AAL, with respect to the benchmark, decreases as the inclusion of more assets with complete information increases. While a behaviour similar to the taxonomy-based results is observed, i.e. an initial nonlinear reduction of uncertainty can be seen for lower levels of exposure knowledge, the shift to a linear trend for the \pm 2 standard deviations occurs at an exposure rate of $50 \%$, after which the results seem to have a much lower increase in accuracy. This behaviour is attributed to the attainment of sufficient data in the pool of assets with known information starting at the 50\% exposure rate mark, which allows the adequate training of the machine learning models to predict probabilities of collapse. It is important to note that the same trend is found for the taxonomy-based curves at a $30 \%$ exposure rate, which is a significant difference in the amount of information required to obtain a substantial reduction in uncertainty. This leads to the preliminary conclusion that taxonomy-based approaches have an advantage over machine learning models to assess total direct losses in portfolios with limited information and the latter should only be considered when significant portions of the inventory have complete information.

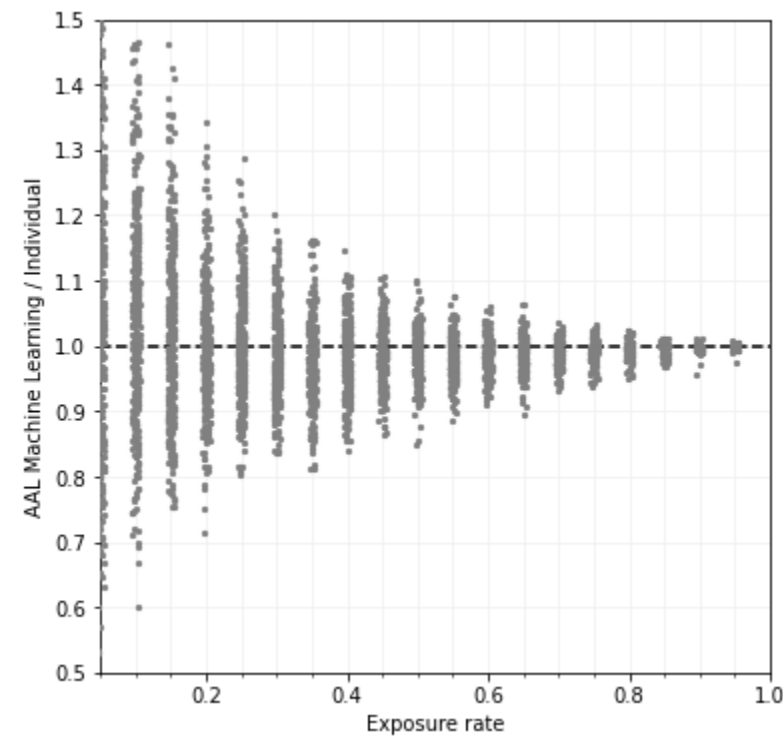

(a)

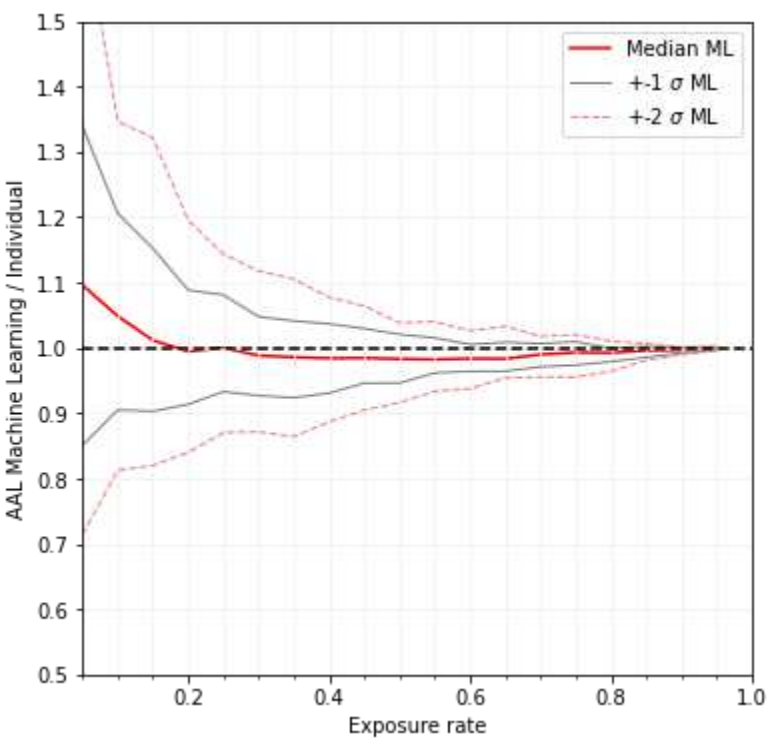

(b)

Figure 18: Results of total inventory direct AAL Machine Learning over Individual based on different exposure knowledge percentages: a) results for each iteration, b) statistical trends observed in results

A comparison between the statistical trends observed in the uncertainty of the calculation of total collapse-based direct AAL using the taxonomy-based approach and the machine learning models is presented in Figure 19 in terms of normalized difference with the benchmark instead of ratios. It can be seen that median values are generally stable when using taxonomy-based curves, even for low exposure rates, while the use of the machine learning models tends to overpredict median losses below the $15 \%$ exposure rate mark. Furthermore, even though similar trends are observed using both methods, the machine learning models definitively outperform the taxonomy-based curves only after achieving an exposure rate of $40 \%$, a point where both methods display an accuracy of approximately $\pm 10 \%$ for the \pm 2 standard deviations in uncertainty. It is important to note that, while this improved comparative performance of machine learning models at the $40 \%$ mark is apparent from the results, the behaviour of 
the uncertainty for \pm 2 standard deviations is still nonlinear at this point, changing to a linear trend until the $50 \%$ exposure rate as mentioned previously. This means that, while it does perform better than the taxonomy-based case, there is still a significant reduction in uncertainty by increasing the exposure knowledge from $40 \%$ to $50 \%$ for the machine learning case. All of this strengthens the conclusion that for cases where low rates of exposure knowledge are present in the inventory, the use of taxonomy-based curves should be preferred to the use of machine learning models.

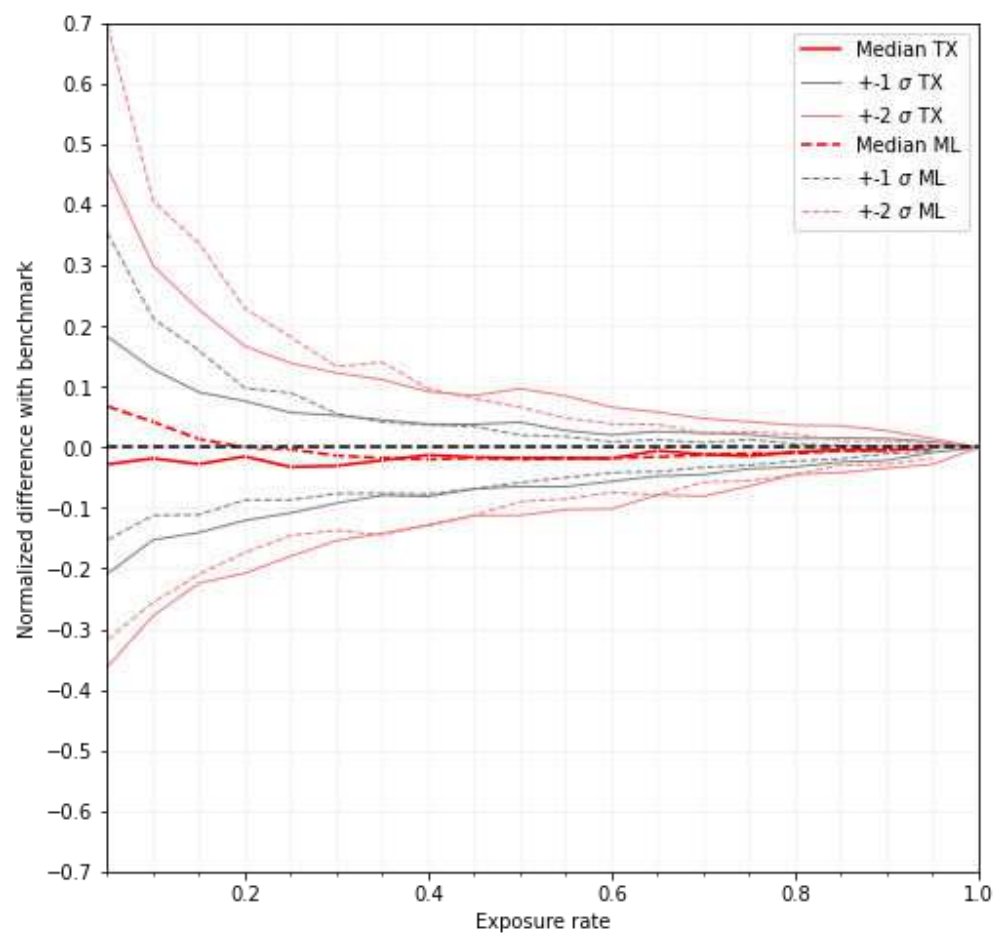

Figure 19: Comparison of results of total inventory direct AAL using a traditional taxonomy-based approach and machine learning models for different exposure knowledge percentages

It is important to note that the machine learning results were obtained using the same model settings for every iteration, independently from taxonomy branch and exposure rate. This represents a limitation in the interpretation of the results since a calibration process for each model would be done in a real case study, which would probably improve the accuracy of the results, making these models potentially more recommendable at lower exposure rates. However, it is also important to mention that the use of machine learning models requires greater expertise and computational resources to build, calibrate, interpret and deploy the algorithms, when compared to the use of taxonomy-based curves. Consequently, analysts should consider the increased effort together with the slight decrease in uncertainty before deciding on a methodology, depending on the available information.

19 In general, for both cases, the presence of a nonlinear behaviour in the reduction of uncertainty in \pm 2 standard deviations when low exposure rates are considered can be used as a decision variable to determine the amount of assets in an inventory that should be analysed. For example, when dealing with the seismic risk assessment of bridge inventories with only $10 \%$ of assets with full knowledge, the cost of increasing the knowledge of the inventory to $20 \%$ could perhaps be justified knowing that, according to the results obtained, this would result in a significant reduction in uncertainty (from $\pm 30 \%$ to $\pm 20 \%$ using taxonomy-based curves). On the other hand, if 50\% of the inventory would have complete information, the same additional cost that would be incurred to increase this value to $60 \%$ would only lead to a reduction in uncertainty from $\pm 10 \%$ to $\pm 8 \%$, when using the same approach. 


\section{Conclusions}

In this study, a database of 308 bridges with complete structural information was used to generate 10 synthetic case studies with different configurations by randomly assigning an asset from the database to each of the 617 locations of bridges in the primary and secondary road networks of the province of Salerno, Italy. The seismic risk level of these case studies, in terms of the total direct average annual losses (AAL) associated to the collapse limit state, was assessed. These results were then used as a benchmark metric to test the implementation of popular and innovative methods in assessing regional bridge portfolios with limited information. For this purpose, the uncertainty that can be expected when considering different percentages of knowledge levels using a taxonomy-based approach as well as a machine learning model was estimated and analysed. Furthermore, the exploration of these results led to the characterization of the trends that can be expected in uncertainty depending on different rates of exposure knowledge, as well as the definition of useful exposure rate thresholds to be used in practical applications to define the amount if exposure information required to obtain a desired level of accuracy in direct loss results.

Based on the results obtained by the application of the different approaches, the following conclusions can be made regarding the epistemic uncertainty that is introduced to regional seismic assessment of bridge portfolios by partial exposure knowledge:

- The use of taxonomy-based curves that average fragility results of assets with similar configurations can lead to accurate median estimates of the aggregate losses over an entire portfolio. This was demonstrated by the results, which show how the median estimates of AAL are consistently close to the benchmark results calculated using structure specific curves, independently from the exposure rate considered;

- When considering the use of taxonomy-based curves, based on the results of this case-study, a complete knowledge of a minimum of $30 \%$ of the inventory is recommended to avoid the large uncertainty associated with lower exposure rates. The same effect was observed when using machine learning models, however the significant reduction in uncertainty happens after $50 \%$ of the inventory is known in this case. Furthermore, the same results showed that, when the known portions of the inventory are larger than these thresholds, incurring in expensive surveying campaigns to increase exposure knowledge becomes less attractive, since it will lead to a lower decrease of uncertainty;

- The use of machine learning algorithms to predict the fragility curves of bridges with incomplete information can outperform typical taxonomy-based approaches only when sufficient results from assets with full information are available to properly train the models. For the case studies explored here, a $40 \%$ exposure rate was found to be the minimum at which the use of machine learning becomes comparatively attractive. However, the results from both approaches are similar and practitioners should consider the added complexity and computer power required to use these models when seeking for an (often slight) increase in performance.

\section{Statements and Declarations}

\subsection{Funding}

The work presented in this paper has been developed within the framework of the project "Dipartimenti di Eccellenza", funded by the Italian Ministry of Education, University and Research at IUSS Pavia. It has also received support from the INFRA-NAT project co-funded by European Commission ECHO Humanitarian Aid and Civil Protection. Project reference: 783298 - INFRA-NAT - UCPM-2017-PP-AG. 


\subsection{Competing interests}

2 The authors have no competing interests to declare that are relevant to the content of this article.

\section{References}

Abarca, A., Monteiro, R., O'Reilly, G. J., Zuccolo, E., \& Borzi, B. (2021). Evaluation of intensity measure performance in the regional assessment of reinforced concrete bridge inventories. Structures and Infrastructures. doi:10.1080/15732479.2021.1979599

Borzi, B., Ceresa, P., Franchin, P., Noto, F., Calvi, G. M., \& Pinto, P. E. (2015). Seismic Vulnerability of the Italian Roadway Bridge Stock. Earthquake Spectra, 31(4), 2137-2161. Retrieved 1 18, 2019, from http://earthquakespectra.org/doi/10.1193/070413eqs190m

Bozorgnia, Y., Abrahamson, N. A., Atik, L. A., Ancheta, T. D., Atkinson, G. M., Baker, J. W., . . . Youngs, R. R. (2014). NGA-West2 Research Project. Earthquake Spectra, 30(3), 973-987. Retrieved 9 21, 2021, from https://earthquakespectra.org/doi/abs/10.1193/072113eqs209m

Chen, R., Branum, D. M., \& Wills, C. J. (2013). Annualized and Scenario Earthquake Loss Estimations for California. Earthquake Spectra, 29(4), 1183-1207. Retrieved 1 12, 2022, from https://earthquakespectra.org/doi/full/10.1193/082911eqs210m

Consiglio dei Ministri . (2003). Primi elementi in materia di criteri generali per la classificazione sismica del territorio nazionale e di normative tecniche per le costruzioni in zona sismica. Roma: G.U. n. 105 del 8 maggio 2003 - S.o. n.72.

D’Ayala, D., Meslem, A., Vamvatsikos, D., Porter, K., Rossetto, T., \& Silva, V. (2015). Guidelines for Analytical Vulnerability Assessment of Low/Mid-Rise Buildings. Pavia: Global Earthquake Model. doi:10.13117/GEM.VULN-MOD.TR2014.12

F.E.M.A. (2013). HAZUS-MH 2.1 Earthquake Model Technical Manual. Washington D.C.: Federal Emergency Management Agency.

Joint Research Centre. (2013). Guidelines for typology definition of European physical assets for earthquake risk assessment. Luxemburg: European Commission.

Kalakonas, P., \& Silva, V. (2021). Seismic vulnerability modelling of building portfolios using artificial neural networks. Earthquake engineering and Structural Dynamics, 1-18. doi:10.1002/eqe.3567

Kohrangi, M., Bazzurro, P., Vamvatsikos, D., \& Spillatura, A. (2017). Conditional spectrum-based ground motion record selection using average spectral acceleration. Earthquake Engineering and Structural Dynamics.

Lin, T., Haselton, C. B., \& Baker, J. W. (2013). Conditional spectrum-based ground motion selection. Part I: Hazard consistency for risk-based assessments. Earthquake Engineering \& Structural Dynamics, 42(12), 1847-1865. Retrieved $7 \quad 23, \quad 2020$, from http://web.stanford.edu/ bakerjw/publications/lin_et_al_(2013)_cs-based_gms_i,_eesd.pdf 
Mangalathu, S., Hwang, S.-H., Choi, E., \& Jeon, J.-S. (2019). Rapid seismic damage evaluation of bridge portfolios using machine learning techniques. Engineering Structures, 201, 109785. Retrieved 9 14, 2021, from https://sciencedirect.com/science/article/pii/s0141029619328068

Mangalathu, S., Soleimani, F., \& Jeon, J. (2017). Bridge classess for regional seismic risk assessment: Improving HAZUS models. Engineering Structures, 755-766. doi:http://dx.doi.org/10.1016/j.engstruct.2017.07.019

Nielson, B. G., \& DesRoches, R. (2007). Analytical Seismic Fragility Curves for Typical Bridges in the Central and Southeastern United States. Earthquake Spectra, 23(3), 615-633. Retrieved 1 12, 2022, from https://earthquakespectra.org/doi/abs/10.1193/1.2756815

OpenStreetMap contributors. (2020). Planet dump. Retrieved from https://planet.osm.org: https://www.openstreetmap.org

O'Reilly, G. (2021). Seismic intensity measures for risk assessment of bridges. Bulletin of Earthquake Engineering, 3671-3699. doi:https://doi.org/10.1007/s10518-021-01114-z

Ozsarac, V., Monteiro, R., \& Calvi, G. (2021). Probabilistic seismic assessment of reinforced concrete bridges using simulated records. Structure and Infrastructure Engineering. doi:10.1080/15732479.2021.1956551

Perdomo, C., Abarca, A., \& Monteiro, R. (2020). Estimation of Seismic Expected Annual Losses for Multi-Span Continuous RC Bridge Portfolios Using a Component-Level Approach. Journal of Earthquake Engineering. doi:10.1080/13632469.2020.1781710

Pitilakis, K., Franchin, P., Khazai, B., \& Wenzel, H. (2014). SYNER-G: Systemic Seismic Vulnerability and Risk Assessment of Complex Urban, Utility, Lifeline Systems and Critical Facilities. Dordrecht: Springer. doi:10.1007/978-94-017-8835-9

Porter, K. A. (2003). An overview of PEER's performance-based earthquake engineering methodology. Ninth International Conference on Applications of Statistics and Probability in Engineering. San Francisco, California.

Silva, V., Crowley, H., Pagani, M., Monelli, D., \& Pinho, R. (2014). Development of the OpenQuake engine, the Global Earthquake Model's open-source software for seismic risk assessment. Natural Hazards, 72(3), 1409-1427. Retrieved 7 23, 2020, from https://link.springer.com/article/10.1007/s11069-013-0618-x

Stefanidou, S. P., \& Kappos, A. J. (2019). Bridge-specific fragility analysis: when is it really necessary? Bulletin of Earthquake Engineering, 17(4), 2245-2280. Retrieved 11 11, 2021, from https://openaccess.city.ac.uk/21190

Woessner, J., Laurentiu, D., Giardini, D., Crowley, H., Cotton, F., Grünthal, G., . . Stucchi, M. (2015). The 2013 European Seismic Hazard Model: key components and results. Bulletin of Earthquake Engineering, 13(12), 3553-3596. Retrieved 9 22, 2021, from https://link.springer.com/article/10.1007/s10518-015-9795-1

Yue, Y., Zonta, D., Bortot, F., \& Zandonini, R. (2010). Assessment of the operation level of a bridge network in post-earthquake scenarios. Retrieved 1 12, 2022, from https://strathprints.strath.ac.uk/55998 
1 Zelaschi, C., Monteiro, R., \& Pinho, R. (2016). Parametric Characterization of RC Bridges for Seismic 2 Assessment Purposes. Structures(7), 14-24.

3 Zuccolo, E., O'Reilly, G., Poggi, V., \& Monteiro, R. (2021). haselREC: an automated open-source 4 ground motion record selection and scaling tool. Bulletin of Earthquake Engineering, 5747-5767. 5 doi:10.1007/s10518-021-01214-w 